\title{
Topological properties of quantum periodic Hamiltonians
}

\author{
Frédéric Faure \\ LPMMC (Maison des Magisteres Jean Perrin, CNRS), BP 166 38042, Grenoble, Cedex 9, France \\ E-mail: faure@labs.polycnrs-gre.fr
}

Received 7 June 1999, in final form 19 October 1999

\begin{abstract}
We consider periodic quantum Hamiltonians on the torus phase space (Harper-like Hamiltonians). We calculate the topological Chern index which characterizes each spectral band in the generic case. This calculation is made by a semiclassical approach with the use of quasimodes. As a result, the Chern index is equal to the homotopy of the path of these quasi-modes on phase space as the Floquet parameter $\theta$ of the band is varied. It is quite interesting that the Chern indices, defined as topological quantum numbers, can be expressed from simple properties of the classical trajectories.
\end{abstract}

\section{Introduction}

Topological quantum numbers are different from quantum numbers based on symmetry because they are insensitive to the imperfections of the systems in which they are observed. In some sense, topological properties are robust properties. Topological quantum numbers have become very important in recent years in condensed matter physics, where measurements of voltage and electrical resistance can be conveniently expressed in terms of them.

If you consider, for example, a simple model of noninteracting electrons moving in a two-dimensional (2D) bi-periodic potential $V(x, y)$ subject to a strong uniform perpendicular magnetic field $B_{z}$ and a low electrical field $E_{x}$, the Hall conductivity $\sigma_{x y}$ of a given filled Landau electronic band turns out to be proportional to an integer $C$ [1]:

$$
\sigma_{x y}=\frac{e^{2}}{h} C
$$

where $C$ is the Chern index of the band, describing the topology of its fibre bundle structure [2-6].

We investigate in this paper the value of $C$ as a function of the potential $V$. In the limit of high magnetic field $B_{z}$, the above model is mapped onto the well known Harper model: the potential $V$ is considered as a perturbation of the cyclotron motion, and the averaging method of mechanics gives an effective Hamiltonian equal to the average of $V$ on the cyclotron circles. We neglect the coupling between the Landau bands [7]. For a high magnetic field (hence for a small cyclotron radius), this transformation gives an effective Hamiltonian $H_{\mathrm{eff}}(q, p) \sim V(q, p)$, which is bi-periodic in position and momentum (the phase space is a 2D torus), and an effective Planck constant $h_{\mathrm{eff}}=h c /(e B)$. In this approximation, trajectories are the contours of $H_{\text {eff }} \sim V$. Furthermore, the expression of $h_{\text {eff }}$ shows that the high magnetic field regime corresponds to the semiclassical limit. This model will be the starting point of our study in the next section. For the sake of simplicity, we will denote $\hbar_{\mathrm{eff}}$ by $\hbar$ in what follows. 
We will restrict ourself to the case where $1 / h=N$ is an integer (but this is not a strong restriction as discussed in section 3.1). The spectrum of these Harper-like models then has a finite band structure. To each band $n=1 \rightarrow N$ is associated a topological Chern index $C_{n}$. In this paper, we calculate all these Chern indices in the semiclassical limit $N \rightarrow \infty$, for a generic Hamiltonian $H(q, p)$ on the torus.

The way we compute these topological indices is as follows. First, we use the fact that the classical dynamics generated by $H$ is integrable, to construct quasi-modes (the usual WKB construction) in section 4. An eigenfunction $\left|\varphi_{n}\left(\theta_{1}, \theta_{2}\right)\right\rangle$ of $\hat{H}$ in band $n$, depends on two quantal parameters related to the periodicity conditions of the wavefunction on the torus. A quasi-mode $\left|\tilde{\psi}_{n}\left(\theta_{1}, \theta_{2}\right)\right\rangle$ is a quantum state localized on a trajectory which is very close to $\left|\varphi_{n}\left(\theta_{1}, \theta_{2}\right)\right\rangle$ in the semiclassical limit $N \rightarrow \infty,[8,9]$ (except when a tunnelling effect occurs).

Because of the tunnelling effect, the eigenfunction $\left|\varphi_{n}\left(\theta_{1}, \theta_{2}\right)\right\rangle$ jumps from one quasimode to another one as $\left(\theta_{1}, \theta_{2}\right)$ are varying and follows a closed path on the torus. By treating correctly this tunnelling effect when it occurs, we obtain a good approximation of the bundle of eigenfunctions $\left|\varphi_{n}\left(\theta_{1}, \theta_{2}\right)\right\rangle$ by quasi-modes $\left|\tilde{\psi}_{n}\left(\theta_{1}, \theta_{2}\right)\right\rangle$, for every value of $\left(\theta_{1}, \theta_{2}\right)$. This gives us the principal result of this paper: the Chern index $C_{n}$ is equal to the homotopy number $I_{n}$ of this path, on the torus, see equation (22). We obtain the Chern indices for the whole range of the spectrum, and show that the noncontractible trajectories play a major role.

This result is based on properties presented in appendix B specifically for the computation of the Chern indices. The analytical methods we use rest on properties of zeros of the Bargmann representation (divisors of a holomorphic section). This appendix is self-contained, and general results presented here could be useful for other purposes where topological Chern indices are involved.

There is a constraint on the global spectrum $[5,6,10]$ : i.e., $\sum_{n} C_{n}=+1$. In section 5 , we give an explanation of this in terms of classical trajectories. It is shown that the noncontractible trajectories on the torus are responsible for this constraint. This nontrivial total Chern number is crucial for explanation of the Hall conductivity [1]. A natural question is then to determine which bands $n$ have a nonzero Chern index $C_{n}$ in the generic case? We answer this question in section 7, where we explain how to compute the Chern indices of the whole spectrum, from the classical Reeb graph.

Our approach is quite similar to that of Thouless et al [1] where they treated $H_{\epsilon}(q, p)=$ $\cos (k q)+\epsilon \cos (p)$. The difference is that they treated $H_{\epsilon}$ within a perturbation approximation (with respect to $\epsilon \ll 1$ ), whereas we use a semiclassical approach. This enables us to treat any generic Hamiltonian $H$, and to describe new examples with nontrivial $C_{n}$, different from the Thouless et al one.

Our results extend previous work of the author $[11,12]$, where the Chern indices were calculated for a restricted range of the spectrum where the classical energy level $\Sigma_{E}=$ $\{(q, p) / H(q, p)=E\}$ consists of two or three contractible trajectories. In these cases, it was shown that $C_{n}$ is zero except for special configurations of the trajectories.

We give a numerical illustration of these results in section 6. It is shown that our results, although derived in the semiclassical limit $N \rightarrow \infty$, are also valid for quite small $N$. This illustrates the robustness of the topological properties. This numerical example is also used throughout the paper to confirm our calculations.

In section 8 , we complete this work by giving a new (but equivalent) derivation of the interpretation of the Chern index $C$ for conductivity, more intuitive than the usual one based on the Kubo formula [1]: we show here that $C$ is equal to the mean velocity of a wavepacket on the torus, when $\left(\theta_{1}, \theta_{2}\right)$ move adiabatically. 


\section{Classical trajectories of a generic Hamiltonian}

A generic classical Hamiltonian on the torus is a generic $C^{\infty}$ Morse function $H(q, p)$ periodic in $q \in \mathbb{R}$ and $p \in \mathbb{R}$, such that

$$
H(q+1, p)=H(q, p+1)=H(q, p) .
$$

In this section we investigate the topological properties of the trajectories of $H$, and introduce the Reeb graph [13], which summarizes them. The aim of this paper is to point out the rules that give the Chern indices from the Reeb graph.

A trajectory $q(t), p(t)$ is a solution of the Hamilton equations of motion:

$$
\begin{aligned}
& \partial_{t} q=\partial_{p} H(q, p) \\
& \partial_{t} p=-\partial_{q} H(q, p) .
\end{aligned}
$$

The trajectories $q(t), p(t)$ evolve on the plane $\mathbb{R}^{2}$, but because of periodicity, they can be considered as trajectories on the torus $T_{q p}$, obtained by identifying the opposite sides of the cell $[0,1] \times[0,1]$.

\subsection{Properties on trajectories}

Because of conservation of energy: $\mathrm{d}(H(q(t), p(t))) / \mathrm{d} t=0$, each trajectory is included in an energy level $\Sigma_{E}=\{(q, p) / H(q, p)=E\}$ of the Morse function $H$. Moreover, the velocity on such a trajectory is never zero, except when there is a critical point of $H$ where $\mathrm{d} H=0$.

We can easily obtain properties on the topology of $\sum_{E}$ :

(1) For noncritical value of $E, \Sigma_{E}$ is a closed one-dimensional curve, and each trajectory can be identified with a connected component of $\Sigma_{E}$. Therefore, each trajectory is a periodic orbit, an oriented closed curve on the torus $T_{q p}$. Its topology (homology) is characterized by two integers $\left(n_{1}, n_{2}\right) \in Z^{2}$ which are the degrees in the $q$ and $p$ directions of $q(t), p(t)$ for $t=0 \rightarrow T$ where $T$ is the period. $\left(n_{1}, n_{2}\right)$ are relatively primes, except if $n_{1}=0$ (or $n_{2}=0$ ) in which case $n_{2}=-1,0$ or +1 . A contractible trajectory has homology type $n_{1}=n_{2}=0$ and is clockwise or anticlockwise.

(2) A generic Hamiltonian has always noncontractible closed trajectories with type $\left(n_{1}, n_{2}\right) \neq(0,0)$. In this case, every other closed trajectory is one of the three types $\left(n_{1}, n_{2}\right),\left(-n_{1},-n_{2}\right)$ or $(0,0)$, and each of these three types exists. (Example: consider a perturbation of $H(q, p)=\cos \left(n_{2} q-n_{1} p\right)$.)

(3) For noncritical value of $E$, each trajectory belongs locally to a family parametrized by the energy $E$ such that every trajectory has the same type. (The critical trajectories are bifurcations between these families.)

For a given generic Hamiltonian, by a good choice of the lattice generators on the plane $(q, p)$, it is possible to deal with trajectories of types $(0,0)(0,+1)$ and $(0,-1)$ only. We will adopt this choice in what follows.

Concerning the critical points and the critical trajectories, there are three sorts of critical points: a local minimum, a saddle point or a local maximum. From Euler's formula ([14] p 29) we have \#(mins) +\#(maxs) $-\#($ saddles $)=0$. (The Euler characteristic of the torus is 0$.

\subsection{Example}

We will now give an example in order to illustrate these statements. This example will be used throughout the paper. 


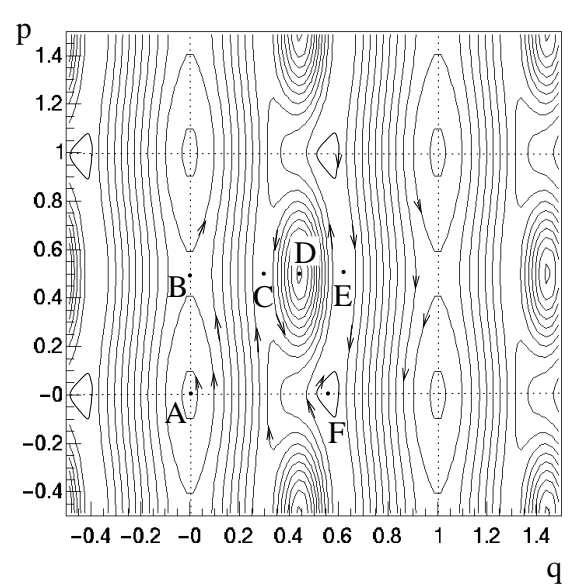

Figure 1. Trajectories and fixed points of Hamiltonian (3).

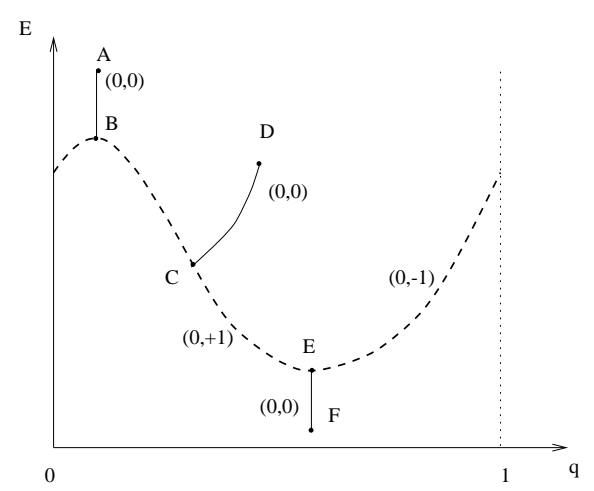

Figure 2. Reeb graph of $H$ : a schematic view of the types of trajectories and bifurcations of Hamiltonian (3). Each point of the dashed curve corresponds to a noncontractible trajectory. They form a one-dimensional family. Each point of a solid curve corresponds to a contractible trajectory.

Consider the following Hamiltonian:

$$
\begin{aligned}
& H(q, p)=H_{0}(q, p)+H_{1}(q, p) \\
& H_{0}(q, p)=\cos (2 \pi q)+0.1 \cos (2 \pi p) \\
& H_{1}(q, p)=P\left[\exp \left(-100\left(q-q_{0}\right)^{2}-10\left(p-p_{0}\right)^{2}\right)\right]
\end{aligned}
$$

where $P$ is a functional operator which sums the function in each cell and makes it periodic:

$$
P[f(q, p)]=\sum_{i, j} f(q+i, p+j) .
$$

The trajectories generated by $H$ are shown in figure 1 , for $q_{0}=0.45, p_{0}=0.45$. There is one minimum $\mathrm{F}$, two maxima $\mathrm{A}, \mathrm{D}$, and three saddle points $\mathrm{B}, \mathrm{C}, \mathrm{E}$.

The figure 2 gives a schematic view of the families of trajectories, with their bifurcations, and homology types. It is called a 'Reeb graph' $\mathcal{R}$ [13]. By definition, it is the topological quotient space $\mathcal{R}=T_{q p} / \mathcal{F}$ where $\mathcal{F}=\left\{\Sigma_{E}\right\}_{E}$ is the set of energy levels. The topology of this graph gives the relative location of the trajectories (the $q$ variable here is only to indicate that the dashed line can be obtained by a section at constant $p$ in figure 1).

The Reeb graph can be drawn for any given generic Hamiltonian. This figure will always have a similar appearance, with one transverse (dashed) curve (from $q=0$ to $q=1$ ) corresponding to the noncontractible trajectories, and branches from it (solid curves), corresponding to contractible trajectories. Critical energies are at the extremities of these branches. The final value of the Chern indices will be read more or less directly from this graph in section 7 .

\section{The quantum Hamiltonian}

In this section we introduce well known material that will be useful in what follows. 


\subsection{The Hilbert space $\mathcal{H}_{P}$ of the plane}

The Hilbert space corresponding to the classical motion in the plane phase space $T^{*}(\mathbb{R}) \cong \mathbb{R}^{2}$ is

$$
\mathcal{H}_{P}=L^{2}(\mathbb{R})
$$

(the index $P$ will remind us that we deal with the plane phase-space).

We choose a symmetric quantization procedure: to the classical Hamiltonian $H(q, p)$ we associate a self-adjoint operator $\hat{H}$ in $\mathcal{H}_{P}$ by

$\hat{H}=\sum_{n_{1}, n_{2} \in Z^{2}} \frac{1}{2} c_{n_{1}, n_{2}} \exp \left(\mathrm{i} 2 \pi n_{1} \hat{q}\right) \exp \left(\mathrm{i} 2 \pi n_{2} \hat{p}\right)+$ Hermitian conjugate

where $c_{n_{1} n_{2}}$ are the Fourier components of the function $H(q, p)$ :

$$
H(q, p)=\sum_{n_{1}, n_{2} \in Z^{2}} c_{n_{1}, n_{2}} \exp \left(\mathrm{i} 2 \pi n_{1} q\right) \exp \left(\mathrm{i} 2 \pi n_{2} p\right) .
$$

Since $H$ is a real-valued function, the complex coefficients $c_{n_{1} n_{2}}$ must satisfy

$$
c_{n_{1}, n_{2}}=\bar{c}_{-n_{1},-n_{2}} \in \mathbb{C} \quad\left(n_{1}, n_{2}\right) \in \mathbb{Z}^{2} .
$$

We denote by $\hat{T}_{Q}$ (resp. $\hat{T}_{P}$ ) the translation operator by one period: $\hat{T}_{Q}$ translates by $Q=1$ a wavefunction $\psi(x)$ and $\hat{T}_{P}$ translates its Fourier transform $\hat{\psi}(p)$ by $P=1$ :

$$
\begin{aligned}
& \hat{T}_{Q} \psi(x)=\psi(x-1) \\
& \hat{T}_{P} \hat{\psi}(p)=\hat{\psi}(p-1) .
\end{aligned}
$$

We may rewrite equations (5) and (6) as

$$
\hat{T}_{Q}=\exp (-\mathrm{i} \hat{p} / \hbar) \quad \hat{T}_{P}=\exp (\mathrm{i} \hat{q} / \hbar) .
$$

Quantum mechanically speaking, the periodicity equation (2) reads

$$
\left[\hat{H}, \hat{T}_{Q}\right]=\left[\hat{H}, \hat{T}_{P}\right]=0 .
$$

To continue, we now have to assume that

$$
\left[\hat{T}_{Q}, \hat{T}_{P}\right]=0 .
$$

It is easy to prove that

$$
\hat{T}_{Q} \hat{T}_{P}=\mathrm{e}^{-\mathrm{i} / \hbar} \hat{T}_{P} \hat{T}_{Q}
$$

hence (8) is fulfilled if and only if there exists an integer $N$ such that

$$
N=\frac{1}{h} \in \mathbb{N}^{*}
$$

with $h=2 \pi \hbar$. This hypothesis (9) can be regarded as a geometric quantization condition, which states that there is an integer number of Planck cells in the phase space. The semiclassical limit $\hbar \rightarrow 0$ corresponds then to the limit $N \rightarrow+\infty$.

We now assume that hypothesis (9) is fulfilled.

Note that this hypothesis is not that restrictive, because any value of $h$ can be well approximated by a rational number $h=m / N$. It is then easy to check that $\left[\hat{T}_{m Q}, \hat{T}_{P}\right]=0$. In this case, we have only then to consider the torus phase space $[0, m Q[\times \mid 0, P[$ instead of $[0, Q[\times \mid 0, P[$. 


\subsection{The Hilbert space $\mathcal{H}_{T}\left(\theta_{1}, \theta_{2}\right)$ for the torus}

According to the commutation relations (7) and (8), the Hilbert space $L^{2}(\mathbb{R})$ may be decomposed as a direct sum of the eigenspaces of the translation operators $\hat{T}_{Q}$ and $\hat{T}_{P}$ :

$$
\begin{aligned}
& \mathcal{H}_{P}=L^{2}(\mathbb{R})=\iint \mathcal{H}_{T}\left(\theta_{1}, \theta_{2}\right) \frac{\mathrm{d} \theta_{1} \mathrm{~d} \theta_{2}}{(2 \pi)^{2}} \\
& \mathcal{H}_{T}\left(\theta_{1}, \theta_{2}\right)=|\psi\rangle \text { such that }\left\{\begin{array}{l}
\hat{T}_{Q}|\psi\rangle=\exp \left(\mathrm{i} \theta_{1}\right)|\psi\rangle \\
\hat{T}_{P}|\psi\rangle=\exp \left(\mathrm{i} \theta_{2}\right)|\psi\rangle
\end{array}\right.
\end{aligned}
$$

with $\left(\theta_{1}, \theta_{2}\right) \in\left[0,2 \pi\left[^{2}\right.\right.$ related to the periodicity of the wavefunction under translations by an elementary cell. The space of the parameters $\left(\theta_{1}, \theta_{2}\right)$ also has the topology of a torus, and will be denoted by $T_{\theta}$.

The space $\mathcal{H}_{T}\left(\theta_{1}, \theta_{2}\right)$ is not a subspace of $L^{2}(\mathbb{R})$, the space of physical states; it is a space of distributions in the $x$ representation.

It is well known that $H_{T}\left(\theta_{1}, \theta_{2}\right)$ is finite-dimensional [1]. To see that, let $|\psi\rangle \in \mathcal{H}_{T}\left(\theta_{1}, \theta_{2}\right)$. The Fourier transform of $\psi(x)$ is $\theta_{2}$-Floquet-periodic of period $P$, so $\psi(x)$ is discrete, it is a sum of Dirac distributions supported at points distant from $h=1 / N$ from each other. Moreover, $\psi(x)$ is $\theta_{1}$-periodic, hence $\psi$ is characterized by the $N$ coefficients at the $N$ Dirac distributions supporting points in the interval $q \in[0,1[$.

Explicitly, a basis of $\mathcal{H}_{T}(\vec{\theta})$ is given by $N$ distribution denoted $|j, \vec{\theta}\rangle$ :

$$
\begin{aligned}
& |j, \vec{\theta}\rangle \equiv \psi_{j, \vec{\theta}}(x)=\frac{1}{\sqrt{N}} \sum_{n_{1} \in \mathbb{Z}} \exp \left(-\mathrm{i} n_{1} \theta_{1}\right) \delta\left(x-q_{j}-n_{1}\right) \\
& j=1, \ldots, N \quad \text { with } \quad q_{j}=\frac{1}{N}\left(j+\frac{\theta_{2}}{2 \pi}\right) .
\end{aligned}
$$

Eventually we get

$$
\operatorname{dim}_{\mathbb{C}} \mathcal{H}_{T}\left(\theta_{1}, \theta_{2}\right)=N
$$

Because of equation (7) the Hamiltonian $\hat{H}$ is block-diagonal with respect to the decomposition equation (10). The operator $\hat{H}$ acts on $\mathcal{H}_{T}\left(\theta_{1}, \theta_{2}\right)$ as a $N \times N$ Hermitian matrix, its spectrum $\sigma\left(\theta_{1}, \theta_{2}\right)$ is made of $N$ eigenvalues and $N$ corresponding eigenfunctions:

$$
\hat{H}\left|\varphi_{n}\left(\theta_{1}, \theta_{2}\right)\right\rangle=E_{n}\left(\theta_{1}, \theta_{2}\right)\left|\varphi_{n}\left(\theta_{1}, \theta_{2}\right)\right\rangle \quad n=1, \ldots, N .
$$

For a given level $n$, assuming that $E_{n}\left(\theta_{1}, \theta_{2}\right)$ is never degenerate $\forall \theta$, the energy level $E_{n}\left(\theta_{1}, \theta_{2}\right)$ forms a band as $\left(\theta_{1}, \theta_{2}\right) \in T_{\theta}$ are varying, and the eigenvectors $\left|\psi_{n}\left(\theta_{1}, \theta_{2}\right)\right\rangle$ form a $2 \mathrm{D}$ surface in the quantum states' space. But for a fixed $\left(\theta_{1}, \theta_{2}\right)$, and any $\lambda \in \mathbb{C}, \lambda\left|\psi_{n}\left(\theta_{1}, \theta_{2}\right)\right\rangle$ is also an eigenvector. So for a fixed level $n$, the family of eigenvectors form a complex-linebundle (of fibre $\cong \mathbb{C} \ni \lambda$ ) in the projective space of the bundle $\mathcal{H}_{T} \rightarrow T_{\theta}$. The topology of this line bundle is characterized by an integer $C_{n} \in \mathbb{Z}$, called the Chern index ([15], ch 1, p 139).

The definition of this topological Chern index is presented in section 5.1.

\subsection{The Hilbert space $\mathcal{H}_{C}\left(\theta_{2}\right)$ for the cylinder}

In the following it will be useful to consider an intermediate decomposition of $\mathcal{H}_{P}$ in terms of states periodic in momentum only:

$$
\begin{aligned}
& \mathcal{H}_{P}=L^{2}(\mathbb{R})=\int \mathcal{H}_{C}\left(\theta_{2}\right) \frac{\mathrm{d} \theta_{2}}{2 \pi} \\
& \mathcal{H}_{C}\left(\theta_{2}\right)=\left\{|\psi\rangle \text { such that } \hat{T}_{P}|\psi\rangle=\exp \left(\mathrm{i} \theta_{2}\right)|\psi\rangle\right\} .
\end{aligned}
$$


Each state in $\mathcal{H}_{C}\left(\theta_{2}\right)$ is a sum of Dirac distributions supported at points $q_{n}=\frac{1}{N}\left(n+\frac{\theta_{2}}{2 \pi}\right)$ with $n \in \mathbb{Z}$. The weights belongs to $l^{2}(\mathbb{Z})$.

Conversely:

$$
\mathcal{H}_{C}\left(\theta_{2}\right)=\int \mathcal{H}_{T}\left(\theta_{1}, \theta_{2}\right) \mathrm{d} \theta_{1}
$$

For each $\theta_{2}$, the Hilbert space $\mathcal{H}_{C}\left(\theta_{2}\right)$ is associated with the cylinder phase space denoted $C_{q p}$, where $(q, p) \in \mathbb{R}^{2}$ is identified with $(q, p+1)$.

From a state $\left|\psi_{P}\right\rangle \in \mathcal{H}_{P}=L^{2}(\mathbb{R})$ of the plane, we can construct a state $\left|\psi_{C}\right\rangle \in \mathcal{H}_{C}\left(\theta_{2}\right)$ of the cylinder by the operation

$$
\left|\psi_{C}\left(\theta_{2}\right)\right\rangle=P_{\theta_{2}}\left|\psi_{P}\right\rangle=\sum_{n=-\infty}^{+\infty} \exp \left(-\mathrm{i} n \theta_{2}\right) T_{P}^{n}\left|\psi_{P}\right\rangle .
$$

From a state $\left|\psi_{C}\right\rangle \in \mathcal{H}_{C}\left(\theta_{2}\right)$ of the cylinder, we can construct a state $\left|\psi_{T}\left(\theta_{1}, \theta_{2}\right)\right\rangle \in$ $\mathcal{H}_{T}\left(\theta_{1}, \theta_{2}\right)$ of the torus by the operation

$$
\left|\psi_{T}\left(\theta_{1}, \theta_{2}\right)\right\rangle=P_{\theta_{1}}\left|\psi_{C}\left(\theta_{2}\right)\right\rangle=\sum_{n=-\infty}^{+\infty} \exp \left(-\mathrm{i} n \theta_{1}\right) T_{Q}^{n}\left|\psi_{C}\left(\theta_{2}\right)\right\rangle
$$

\section{The quasi-modes}

\subsection{Construction of a quasi-mode}

The WKB method allows the construction of a quasi-mode for integrable dynamics (see [8] and [9] p 235).

First, we recall the definition and general properties of quasi-modes.

Definition 1. If $\Gamma$ is a given closed trajectory with energy $E$, a quasi-mode with error $\epsilon$, is a quantum state $|\tilde{\psi}\rangle$ localized near $\Gamma$, which satisfies

$$
\| \hat{H}|\tilde{\psi}\rangle-\tilde{E}|\tilde{\psi}\rangle \|=\epsilon=\mathrm{o}\left(h^{\infty}\right)
$$

with $h=1 / N$ going to zero, with $(E-\tilde{E})=\mathrm{o}(h)$. The trajectory $\Gamma$ is called the support of $|\tilde{\psi}\rangle$ and is denoted

$$
\Gamma=\operatorname{Supp}(|\tilde{\psi}\rangle)
$$

If we want to improve this error $\epsilon$ (and have an error exponentially small with respect to $h$ ), we have to take into account the tunnelling effect between $\Gamma$, and $\Gamma^{\prime}$ the 'closest' trajectory $\Gamma^{\prime}$ which has the same energy $E$ [16-18]. We will return to this point later.

Proposition 1 ([9]). The interval $[\tilde{E}-\epsilon, \tilde{E}+\epsilon]$ contains at least one eigenvalue of $\hat{H}$.

Let $\Delta_{\alpha}=[\tilde{E}-\alpha, \tilde{E}+\alpha]$. If $\Delta_{\alpha}$ contains only one eigenvalue $E^{*}$, with eigenvector $|\varphi\rangle$, then (for normalized vectors)

$$
\exists \beta \in R \quad \||\varphi\rangle-\mathrm{e}^{\mathrm{i} \beta}|\tilde{\psi}\rangle \| \leqslant \frac{\epsilon}{\alpha}
$$

consequently, $\langle\varphi \mid \tilde{\psi}\rangle \neq 0$ as soon as $\epsilon \leqslant \alpha / 2$ (for our purpose, we will say in that case, that $|\tilde{\psi}\rangle$ is a 'good approximation' of $|\varphi\rangle$. This condition will be sufficient to construct a bundle of quasi-modes with the same topology (the same Chern index).

Let us now give properties of quasi-modes specific to our problem. The explicit construction of the quasi-modes can be found in [19-21]. 


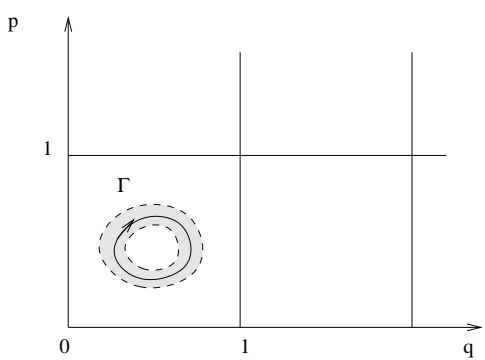

Figure 3. Quasi-mode localized on a trajectory $\Gamma$ of type $(0,0)$ in cell $(0,0)$.

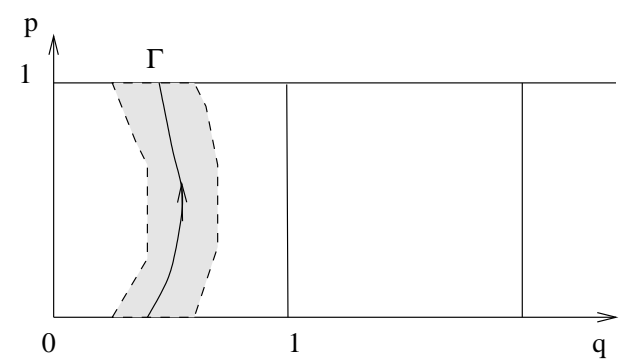

Figure 4. Quasi-mode localized on a trajectory $\Gamma$ of type $(0,+1)$, in cell $(0)$ on the cylinder.

Contractible trajectories of type $(0,0)$. For a noncritical contractible trajectory $\Gamma$ of type $(0,0)$, we first construct a quasi-mode on the plane, $\left|\tilde{\psi}_{P}\right\rangle \in \mathcal{H}_{P}$. From the usual WKB construction, this quasi-mode is localized on a single image of $\Gamma$, for example in the cell $(0,0)$. See figure 3 .

The energy $\tilde{E}$ of this quasi-mode is given by the usual EBK condition:

$$
S(\tilde{E})=(k+1 / 2) h+\mathrm{o}(h) \quad k \in \mathbb{Z}
$$

with $S(\tilde{E})$ the surface inclosed in the trajectory $\Gamma$.

A quasi-mode $\left|\tilde{\psi}_{C}\left(\theta_{2}\right)\right\rangle$ on the cylinder can be deduced by equation (12). A quasi-mode $\left|\tilde{\psi}_{T}\left(\theta_{1}, \theta_{2}\right)\right\rangle$ on the torus can be constructed by equation (13). Note that the energy of these quasi-modes $\tilde{E}$ does not depend on $\left(\theta_{1}, \theta_{2}\right)$.

Noncontractible trajectories of type $(0, \pm 1)$. If $\Gamma$ is a noncritical trajectory with type $\vec{n}=(0, \pm 1), \Gamma$ is closed on the cylinder $C_{q p}$ and a quasi-mode $\left|\tilde{\psi}_{C}\left(\theta_{2}\right)\right\rangle \in \mathcal{H}_{C}\left(\theta_{2}\right)$ can be constructed for every $\theta_{2}$.

$\left|\tilde{\psi}_{C}\left(\theta_{2}\right)\right\rangle$ is localized on $\Gamma$, for example in cell 0: see figure 4 .

The energy $\tilde{E}$ of this quasi-mode is given by the condition

$$
S(\tilde{E})=\left(k-\theta_{2} / 2 \pi\right) h+\mathrm{o}(h) \quad k \in \mathbb{Z}
$$

where $S(\tilde{E})$ is the surface included in the unit cell, on the right of the trajectory $\Gamma$ (here oriented by increasing $p$ ). We justify this condition in the next paragraph.

Note that the energy $\tilde{E}\left(\theta_{2}\right)$ as well as the support $\Gamma\left(\theta_{2}\right)$ depend continuously on $\theta_{2}$.

A quasi-mode $\left|\tilde{\psi}_{T}\left(\theta_{1}, \theta_{2}\right)\right\rangle$ on the torus can be deduced from $\left|\tilde{\psi}_{C}\left(\theta_{2}\right)\right\rangle$ by equation (13).

For contractible and noncontractible trajectories, another quasi-mode with the same energy can be constructed in the cell $n$ by

$$
\left|\tilde{\psi}_{C, n}\left(\theta_{2}\right)\right\rangle=T_{Q}^{n}\left|\tilde{\psi}_{C}\left(\theta_{2}\right)\right\rangle
$$

From the WKB construction, the error $\epsilon$ of these quasi-modes is of $\mathrm{o}\left(h^{\infty}\right)$. The error $\epsilon$ is due to tunnelling interaction with 'the nearest quasi-mode of nearby energy' (see the next section).

Critical trajectories. If $E_{0}$ is a critical energy, quasi-modes of energy near $E_{0}$ (in the interval $\left.\left[E_{0} \pm \mathrm{O}(h)\right]\right)$ and localized near the critical trajectory, can be constructed. See [22-24].

Justification of quantization rule equation (15). When constructing a WKB quasi-mode on a closed trajectory, the quantization rule comes from the phase matching. The phase 


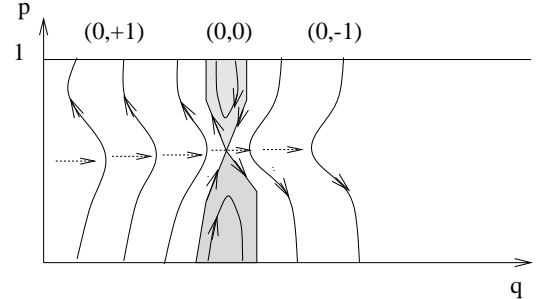

Figure 5. The dashed arrows indicate the motion of the support of a quasi-mode, $\theta_{2} \rightarrow \Gamma\left(\theta_{2}\right)$, over all noncontractible trajectories, as $\theta_{2}$ increases.

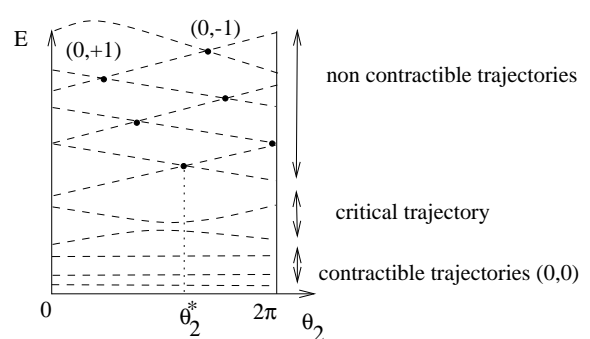

Figure 6. The energy of the quasi-modes as a function of $\theta_{2}$.

of the Fourier transform of the quasi-mode evolves like $\left(-\int q \mathrm{~d} p\right)$ along the trajectory $q(t), p(t)$. For a trajectory $\Gamma$ of type $(0, \pm 1)$, the phase accumulated over a period is then $\varphi=S / \hbar=-\int_{\Gamma} q \mathrm{~d} p / \hbar$, where $S$ is the surface on the right of the trajectory $\Gamma$ (oriented by increasing value of $p$ ). The periodicity condition (10) requires that $\varphi=\theta_{2}[2 \pi]$. This gives the result (15).

\subsection{The semiclassical spectrum without tunnelling corrections}

We now investigate the global properties of the quasi-modes, with respect to $\theta_{2}$.

Consider fixed values of $\left(\theta_{1}, \theta_{2}\right) \in T_{\theta}$. A WKB construction as previously described, gives us $N$ quasi-modes $\left|\tilde{\psi}_{T}\left(\theta_{1}, \theta_{2}\right)\right\rangle_{n}$ with energies $\tilde{E}_{n}\left(\theta_{1}, \theta_{2}\right)$ over the whole energy range, with $n=1 \rightarrow N$. This is a semiclassical approximation $\sigma_{s c}\left(\theta_{1}, \theta_{2}\right)$ of the spectrum $\sigma\left(\theta_{1}, \theta_{2}\right)$ defined by equation (11). In fact, the construction was made on $\mathcal{H}_{C}\left(\theta_{2}\right)$ and does not depend on $\theta_{1}$, so we will note it $\sigma_{s c}\left(\theta_{2}\right)$. (For contractible trajectories, it does not even depend on $\theta_{2}$.)

From the above construction, each quasi-mode $\left|\tilde{\psi}_{C}\left(\theta_{2}\right)\right\rangle$ depends continuously on $\theta_{2}$. So each quasi-mode belongs to a function $\Psi: \theta_{2} \rightarrow\left|\tilde{\psi}_{C}\left(\theta_{2}\right)\right\rangle \in \mathcal{H}_{C}\left(\theta_{2}\right)$ which is periodic with respect to $\theta_{2}$ (up to a phase), with a period $\Theta_{2}=2 \pi m$, with $m \in N$. (Otherwise, if the function $\Psi$ were not periodic, the spectrum $\sigma_{s c}\left(\theta_{2}\right)$ would be infinite for a fixed value of $\theta_{2}$.) The corresponding energy $\tilde{E}\left(\theta_{2}\right)$ and the support $\Gamma\left(\theta_{2}\right)$ are therefore also periodic with the same periods.

If the trajectory $\Gamma$ is contractible (type $(0,0)$ ), the energy $\tilde{E}$ and the support $\Gamma$ are fixed. They do not depend on $\theta_{2}$, and the period is $\Theta_{2}=2 \pi$.

For a noncontractible and noncritical trajectory (type $(0, \pm 1)$ ), equation (15) tells that the surface $\theta_{2} \rightarrow S\left(\theta_{2}\right)$ on the right of $\Gamma$ is a strictly decreasing function because $\mathrm{d} S / \mathrm{d} \theta_{2}=$ $-h / 2 \pi$. Then $\theta_{2} \rightarrow \Gamma\left(\theta_{2}\right)$ moves from the left to the right on the set of noncontractible trajectories on the cylinder $C_{q p}$. This function reaches all the noncontractible trajectories, and jumps over a connected component of contractible trajectories, via a critical point: see figure 5 .

The surface $S\left(\theta_{2}\right)$ is always a decreasing function, but the corresponding energy $E\left(\theta_{2}\right)$ decreases for trajectories of type $(0,+1)$ and increases for type $(0,-1)$, with the behaviour shown in figure 2 , if we plot $E\left(\theta_{2}\right)$ for $\theta_{2}=0 \rightarrow 2 \pi m$. Figure 6 shows this function $E\left(\theta_{2}\right)$, but folded in the interval $\theta_{2}=0 \rightarrow 2 \pi$.

So we obtain the following proposition.

Proposition 2. The global spectrum $\theta_{2} \rightarrow \sigma_{s c}\left(\theta_{2}\right)$ is the union of one single function $\Psi_{n c}: \theta_{2} \rightarrow\left|\tilde{\psi}_{n c}\left(\theta_{2}\right)\right\rangle \in \mathcal{H}_{C}\left(\theta_{2}\right)$, and many others $\left(\Psi_{c}\right)_{i=1, \ldots, N_{c}}: \theta_{2} \rightarrow\left|\tilde{\psi}_{c}\left(\theta_{2}\right)\right\rangle \in \mathcal{H}_{C}\left(\theta_{2}\right)$ such that: the support $\Gamma_{n c}$ of $\Psi_{n c}$ is on the noncontractible trajectories. The period of $\Psi_{n c}$ is 


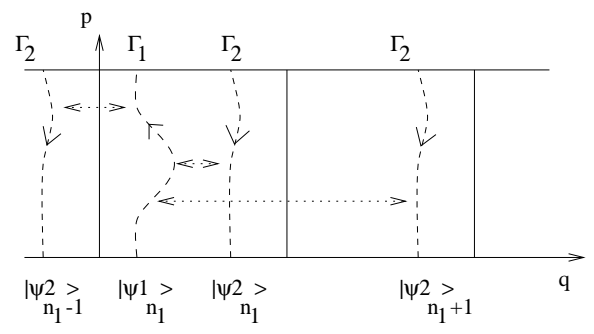

Figure 7. Tunnelling effect between quasi-mode $\left|\psi 1_{n 1}\right\rangle$ on trajectory $\Gamma_{1}$, in cell $n_{1}$, and quasi-modes $\left|\psi 2_{n 2}\right\rangle$. We keep only the dominant tunnelling interaction, here due to $\left|\psi 2_{n 1}\right\rangle$.

$\Theta_{2}=2 \pi \cdot N_{n c}$, such that $N_{n c} \cdot h \sim S_{n c}$ the surface occupied by the noncontractible trajectories. In phase space, this support $\theta_{2} \rightarrow \Gamma_{n c}\left(\theta_{2}\right)$ is a closed cycle of homotopy 1 on the cylinder $C_{q p}$.

The support $\Gamma_{c}$ of each function $\left(\Psi_{c}\right)_{i}$ is on a fixed contractible trajectory. Its period is $\Theta_{2}=2 \pi$. The number $N_{c}$ of these functions is such that $N_{c} \cdot h \sim S_{c}$ the surface occupied by the contractible trajectories. Note that $N=N_{c}+N_{n c}$ and $1=S_{n c}+S_{c}$.

We now discuss the error of the spectrum $\sigma_{s c}\left(\theta_{2}\right)$.

The error of each quasi-mode is $\epsilon \sim \mathrm{o}\left(h^{\infty}\right)$. There are crossings in the spectrum $\sigma_{s c}\left(\theta_{2}\right)$ for discrete values $\theta_{2}^{*}$, (caused by the noncontractible function when $\tilde{E}_{n c}\left(\theta_{2}\right)=\tilde{E}_{n c}\left(\theta_{2}^{\prime}\right)$ or $\tilde{E}_{n c}\left(\theta_{2}\right)=\tilde{E}_{c}\left(\theta_{2}^{\prime}\right)$ ), see figure 6 . For fixed $\theta_{2}$, away from a small neighbourhood of every crossing value $\theta_{2}^{*}$, the spectrum $\sigma_{s c}\left(\theta_{2}\right)$ has $N$ discrete eigenvalues and each eigenvalue is isolated from the overs by an interval of length $\alpha \gg \epsilon$. We deduce from properties 1 that the corresponding quasi-modes $\left|\tilde{\psi}_{n}\left(\theta_{2}\right)\right\rangle$ (in fact their image in $\mathcal{H}_{T}$ by the mapping $P_{\theta_{2}}$ ), are a good approximation of the actual eigenvectors $\left|\varphi_{n}\left(\theta_{1}, \theta_{2}\right)\right\rangle$, for any $\theta_{1}$.

Let us remark that this semiclassical spectrum $\sigma_{s c}\left(\theta_{2}\right)$ does not depend on $\theta_{1}$ and is thus infinitely degenerate. This correspond to the invariance by the translation (16). This is not the case in the actual spectrum $\sigma\left(\theta_{1}, \theta_{2}\right)$ because of tunnelling effect.

Figure 9 shows the numerical spectrum of our example $H(q, p)$, equation (3). From this, we deduce what the semiclassical spectrum should look like: figure (10). Compare this with the semiclassical spectrum, figure 6.

$\sigma_{s c}\left(\theta_{2}\right)$ is not a good approximation of the actual spectrum near the crossing values $\theta_{2}^{*}$. To improve this, in the next section, we will take into account the tunnelling effect at each crossing of energy. It causes a splitting of levels and we will recover $N$ well defined bands of energy.

\subsection{The semiclassical spectrum with tunnelling corrections}

Consider a neighbourhood $U$ of a value $\theta_{2}^{*}$, where there is a crossing in the spectrum $\sigma_{s c}\left(\theta_{2}\right)$ between two energies: $\tilde{E}_{1}\left(\theta_{2}^{*}\right)=\tilde{E}_{2}\left(\theta_{2}^{*}\right)$. See figure 6 . As said before, this crossing is caused by two noncontractible trajectories when $\tilde{E}_{n c}\left(\theta_{2}\right)=\tilde{E}_{n c}\left(\theta_{2}^{\prime}\right)$ or by one noncontractible trajectory and one contractible one: $\tilde{E}_{n c}\left(\theta_{2}\right)=\tilde{E}_{c}$. The crossing due to two contractible trajectories $\tilde{E}_{c 1}=\tilde{E}_{c 2}$ is not considered here, because it does not occur for a generic Hamiltonian $H$. See $[11,12]$ for results treating this nongeneric case. From equation (16), these energies correspond to two families of quasi-modes: $\left|\tilde{\psi} 1_{C, n_{1}}\right\rangle,\left|\tilde{\psi} 2_{C, n_{2}}\right\rangle \in \mathcal{H}_{C}\left(\theta_{2}\right)$ where $n_{1} \in \mathbb{Z}, n_{2} \in \mathbb{Z}$. We suppose that these quasi-modes are localized, respectively, on two trajectories $\Gamma_{1}$ and $\Gamma_{2}$, in cells $n_{1}$ and $n_{2}$.

To improve the error $\epsilon$ of the quasi-mode say $\left|\tilde{\psi} 1_{C, n_{1}}\right\rangle$, we have to take into account the tunnelling interactions with other quasi-modes which have nearby energy $\left|\tilde{\psi} j_{C, m}\right\rangle$ with $j=1,2$ and $m \in \mathbb{Z}$ : see figure 7 . This tunnelling interaction comes from nonvanishing terms $A_{j, m}=\left\langle\tilde{\psi} 1_{C, n_{1}}|\hat{H}| \tilde{\psi} j_{C, m}\right\rangle$. We will treat the tunnelling effect at the leading order, by 
keeping only the dominant term. Consider first the terms $A_{2, n_{2}}$ with $j=2$, and $n_{2} \in \mathbb{Z}$. We keep the $n_{2}$ term with greatest modulus: $\left|A_{2, n_{2}}\right|$. This term is $\mathrm{o}\left(h^{\infty}\right)$ and describes the tunnelling interaction between $\left|\tilde{\psi} 1_{C, n_{1}}\right\rangle$ and the 'nearest' quasi-mode $\left|\tilde{\psi} 2_{C, n_{2}}\right\rangle$ : see figure 7 . It is clear that the nearest quasi-mode is $n_{2}=n_{1}$ or $n_{2}=n_{1} \pm 1$, because other values of $n_{2}$ are in more distant cells. The other terms are $\mathrm{o}\left(h^{\infty}\right)$ with respect to the leading order term. Consider now the terms $A_{1, m}$ with $j=1$, and $m \in \mathbb{Z}$. The dominant term is for $m=n_{1}$ : $A_{1, n_{1}}=\left\langle\tilde{\psi} 1_{C, n_{1}}|\hat{H}| \tilde{\psi} 1_{C, n_{1}}\right\rangle=\tilde{E}_{1}\left(\theta_{2}\right)$. This is the energy of the quasi-mode. The other terms, which describe tunnelling interaction between $\left|\tilde{\psi} 1_{C, n_{1}}\right\rangle$ and its images $\left|\tilde{\psi} 1_{C, m}\right\rangle$ in other cells, are $\mathrm{o}\left(h^{\infty}\right)$ with respect to $\left|A_{2, n_{2}}\right|$ and so are negligible.

We have obtained that the dominant correction to the quasi-modes $\left|\tilde{\psi} 1_{C, n_{1}}\right\rangle$ is described by the $2 \times 2$ tunnelling interaction matrix:

$$
A\left(\theta_{2}\right)=\left(\left\langle\tilde{\psi} i_{C, n_{1}}|\hat{H}| \tilde{\psi} j_{C, n_{2}}\right\rangle\right)_{i, j}=\left(\begin{array}{cc}
\tilde{E}_{1}\left(\theta_{2}\right) & A_{2, n_{2}}\left(\theta_{2}\right) \\
\hline A_{2, n_{2}}\left(\theta_{2}\right) & \tilde{E}_{2}\left(\theta_{2}\right)
\end{array}\right)
$$

where $\left|\tilde{\psi} 2_{C, n_{2}}\right\rangle$ is the 'nearest' quasi-mode to $\left|\tilde{\psi} 1_{C, n_{1}}\right\rangle, n_{2}=n_{1}$ or $n_{2}=n_{1} \pm 1$, and $\left|A_{2, n_{2}}\right|$ is $\mathrm{o}\left(h^{\infty}\right)$.

By diagonalizing the matrix $A$, we obtain two quasi-modes $\left|\tilde{\psi}_{C+}\left(\theta_{2}\right)\right\rangle$ and $\left|\tilde{\psi}_{C-}\left(\theta_{2}\right)\right\rangle \in$ $\mathcal{H}_{C}\left(\theta_{2}\right)$ expressed as a linear superposition of $\left|\tilde{\psi} 1_{C, n_{1}}\right\rangle$ and $\left|\tilde{\psi} 2_{C, n_{2}}\right\rangle$, and two energies $\left.E_{+}\left(\theta_{2}\right)\right\rangle E_{-}\left(\theta_{2}\right)$ with a gap $\left|\tilde{E}_{+}\left(\theta_{2}\right)-\tilde{E}_{-}\left(\theta_{2}\right)\right| \geqslant\left|A_{2, n_{2}}\right|$. But the error of the quasi-modes is $\mathrm{o}\left(h^{\infty}\right)$ with respect to this gap. From property (1), we deduce that these quasi-modes $\left|\tilde{\psi}_{C \pm}\left(\theta_{2}\right)\right\rangle$ (their image in $\mathcal{H}_{T}$, actually) are a good approximation of the eigenvectors $\left|\varphi_{i}\left(\theta_{1}, \theta_{2}\right)\right\rangle$, for any $\theta_{1}$.

Now if we match these results with the outside of the neighbourhood $U$ of $\theta_{2}^{*}$, we can obtain a continuous dependence of $\left|\tilde{\psi}_{C}\left(\theta_{2}\right)\right\rangle$ with respect to $\theta_{2} \in \mathbb{R}$.

Proposition 3. We have obtained quasi-modes $\left|\tilde{\psi}_{C}\left(\theta_{2}\right)\right\rangle$ on the cylinder, which are a good approximation of the spectrum equation (11) for any $\theta_{1}, \theta_{2}$ in the sense that

$$
\left\langle\varphi_{n}\left(\theta_{1}, \theta_{2}\right) \mid \tilde{\psi}_{T}\left(\theta_{1}, \theta_{2}\right)\right\rangle \neq 0 \quad \text { for every } \quad\left(\theta_{1}, \theta_{2}\right) .
$$

We note this approximate spectrum by $\sigma_{s c, t}\left(\theta_{2}\right)$ (the energies do not depend on $\theta_{1}$ ).

There is no more crossing in the energy levels, so we have $N$ well defined (semiclassical) bands and corresponding quasi-modes $\left|\tilde{\psi}_{C, n}\left(\theta_{2}\right)\right\rangle, n=1, \ldots, N$.

At each avoided crossing, we have obtained that the support of a quasi-mode jumps from the trajectory $\Gamma_{1}$ in cell $n_{1}$ (resp. $\Gamma_{2}$ in $n_{2}$ ) to the closest trajectory $\Gamma_{2}$ in cell $n_{2}=n_{1}$ or $n_{2}=n_{1} \pm 1$ (resp. $\Gamma_{1}$ in $\left.n_{1}\right)$ as in figure 7 .

More globally, for each band $n$, we derived a function for the support of the quasi-mode $\left|\tilde{\psi}_{C, n}\left(\theta_{2}\right)\right\rangle$ on the cylinder $C_{q p}$ :

$$
S_{C n}: \theta_{2} \in R \rightarrow \operatorname{Supp}\left(\left|\psi_{C, n}\left(\theta_{2}\right)\right\rangle\right) \subset C_{q p}
$$

The image $S_{T, n}$ of $S_{C, n}$ on the torus is periodic for $\theta_{2}=0 \rightarrow 2 \pi$, and $S_{C, n}$ is a lifting of $S_{T, n}$ on the cylinder. With $S_{C, n}$ is associated a homotopic number $I_{n} \in \mathbb{Z}$. Precisely, $I_{n}$ is given by

$$
T_{Q}^{I_{n}}\left[\operatorname{Supp}\left(\left|\psi_{C, n}(0)\right\rangle\right)\right]=\left[\operatorname{Supp}\left(\left|\psi_{C, n}(2 \pi)\right\rangle\right)\right] .
$$

$I_{n}$ is simply the result of the jumps of the quasi-mode from cell $n_{1}$ to cell $n_{1}+I_{n}$ as $\theta_{2}=0 \rightarrow 2 \pi$. 


\section{Chern indices}

\subsection{Definition}

The eigenfunctions $\left|\varphi_{n}\left(\theta_{1}, \theta_{2}\right)\right\rangle$ of the band $n$, equation (11), form a complex line fibre bundle over the torus $\left(\theta_{1}, \theta_{2}\right) \in T_{\theta}$. On a contractible subset of $U \subset T_{\theta}$ we can choose the states $\left|\varphi_{n}\left(\theta_{1}, \theta_{2}\right)\right\rangle$ such that they are normalized and that they form a (continuous) section over $U$. Note that this is not possible over the whole torus $T_{\theta}$ except if the bundle is trivial.

The global topology of this complex line bundle is characterized by its Chern index ([15] p 139, [25]).

Because of the natural Hilbert scalar product on $L^{2}(\mathbb{R})=\iint \mathcal{H}_{T}\left(\theta_{1}, \theta_{2}\right) \mathrm{d} \theta_{1} \mathrm{~d} \theta_{2}$, which induces the Berry (or Chern) connection $[2,26]$, this topological number is explicitly given by the integral of the Berry (or Chern) curvature ([1], [15] p 141):

$$
C_{n}=\frac{\mathrm{i}}{2 \pi} \int_{T_{\theta}}\left(\left\langle\partial_{\theta_{1}} \varphi_{n} \mid \partial_{\theta_{2}} \varphi_{n}\right\rangle-\left\langle\partial_{\theta_{2}} \varphi_{n} \mid \partial_{\theta_{1}} \varphi_{n}\right\rangle\right) \mathrm{d} \theta_{1} \mathrm{~d} \theta_{2}
$$

obtained by summing over local open subsets $U_{i}$ which covered $T_{\theta}$, and sections chosen in each of them.

This expression has been used intensively for our numerical calculations.

Moreover, it can be shown (see e.g. $[6,10]$ ) that

$$
\sum_{n=1}^{N} C_{n}=1
$$

There is an alternative expression for $C_{n}$ more suitable for our analytical calculations, given in equation (27), (see [15] p 141). It is based on the motion of the zeros of the Bargmann representation of the states $\left|\varphi_{n}\left(\theta_{1}, \theta_{2}\right)\right\rangle$.

\subsection{Chern indices of the semiclassical bands}

Our aim is to express the index $C_{n}$ from the description of the classical dynamics.

Because of property (17), we deduce from theorem 1 (appendix B), that the band of the semiclassical spectrum $\sigma_{s c, t}$ have the same topology (and same Chern indices) as the actual energy bands.

Our work consists now in computing the index $C_{n}$ of the semiclassical band $n$.

From theorem 6 (appendix B), the result is simply that

$$
C_{n}=I_{n}
$$

where $I_{n}$ defined by equation (19), is the homotopic number characterizing the path followed by the support of the quasi-mode on the cylinder, when $\theta_{2}$ is varying from 0 to $2 \pi$.

\subsection{Global analysis of the Chern indices}

In this section we calculate the sum of Chern indices for consecutive bands, in order to recover the result, equation (21), within our semiclassical approach.

For each band $n=1 \rightarrow N$, we have defined the cycle of the support $S_{T, n}$ on the torus $T_{q p}$, equation (18). The last result, equation (22), is that the Chern index $C_{n}=I\left(S_{n}\right)$ is the homotopy number of this cycle (in the $q$ direction). (We will now drop the $T$ suffix in $S_{n}$.)

We decide to define the sum of two or more consecutive cycles $S_{n}+S_{n+1}+\cdots+S_{n+a}$ by removing the jumping due to tunnelling at the crossings between two consecutive cycles $S_{i}$ and $S_{i+1}$. This is illustrated in figure 8. The homotopy is then $I\left(S_{n}+\cdots+S_{n+a}\right)=$ $I\left(S_{n}\right)+\cdots+I\left(S_{n+a}\right)$, because the removing is a local operation. 

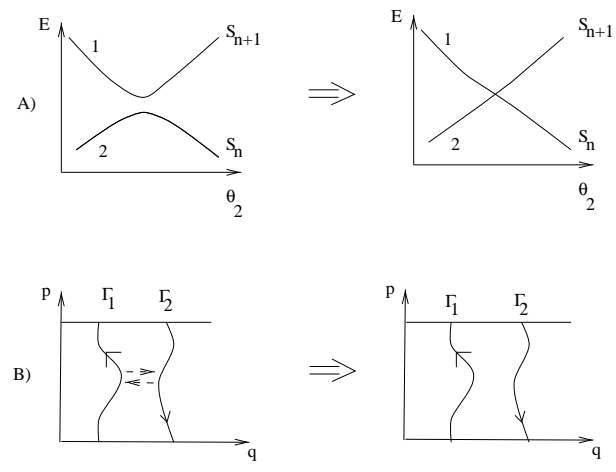

Figure 8. The tunnelling effect generates a splitting between energy levels (A), and a jump of quasi-modes between trajectories (B). In order to calculate the sum $\sum_{n=1}^{N} C_{n}$, we have to remove this splitting, as well as this jump.

$\sum_{n=1}^{N} S_{n}$ is then the support of the semiclassical spectrum $\sigma_{s c}$ without tunnelling, and from proposition (2), it is composed of one cycle $\Gamma_{n c}$ of homotopy 1 and many constant cycles $\Gamma_{c, i}$ of homotopy 0 . This gives

$$
\sum_{n=1}^{N} C_{n}=\sum_{n=1}^{N} I\left(S_{n}\right)=I\left(\sum_{n=1}^{N} S_{n}\right)=+1 .
$$

We recover equation (21). The total Chern index $(+1)$ is interpreted here as the motion of the noncontractible quasi-mode on the torus as $\theta_{2}$ is varying (in accordance with theorem 6 , appendix B).

\section{Numerical illustration}

We will illustrate the above results on the Hamiltonian equation (3). The numerical calculations of the Chern indices have been done with the curvature formula equation (20). For $N=11$ levels, the Chern indices are

\begin{tabular}{ccccc}
\hline$C_{1 \rightarrow 4}$ & $C_{5}$ & $C_{6}$ & $C_{7}$ & $C_{8 \rightarrow 11}$ \\
\hline 0 & +1 & -1 & +1 & 0 \\
\hline
\end{tabular}

Our analytical results have been obtained in the $N \rightarrow \infty$ limit. Although, $N=11$ is not very high, the numerical results which follow can well be interpreted in the semiclassical description of this paper.

The minimum value of $N$ required to get correct semiclassical estimates can be evaluated in the following manner: the mesh provided by Planck cells of magnitude $1 / N$ has to be about so fine that all phase space structures in a plot like figure 1 can be resolved.

Figure 9 shows the energy levels $E_{n}\left(\theta_{1}, \theta_{2}\right)$ for $n=1 \rightarrow 11$, as a function of $\theta_{2}$. The dependence on $\theta_{1}$ gives a width of the levels, but is very weak and not visible in the figure.

In this energy spectrum, one can clearly distinguish two categories of energy levels. These two categories are reproduced in figure 10, where we have artificially dropped the splittings. This figure corresponds to the semiclassical spectrum $\sigma_{s c}\left(\theta_{2}\right)$.

There are four energy levels (solid curves) which do not depend strongly on $\theta_{2}$ and have periodicity $2 \pi$. In the light of proposition (2), these energy levels correspond to four quasimodes $\left|\tilde{\psi}_{c}\left(\theta_{2}\right)\right\rangle$ localized on contractible trajectories. There is also one continuous energy level $E_{n c}\left(\theta_{2}\right)$ (dashed curves) which depends on $\theta_{2}$ and has periodicity $2 \pi m$ with $m=7$. This energy level corresponds to quasi-modes $\left|\tilde{\psi}_{n c}\left(\theta_{2}\right)\right\rangle$ localized on noncontractible trajectories. 


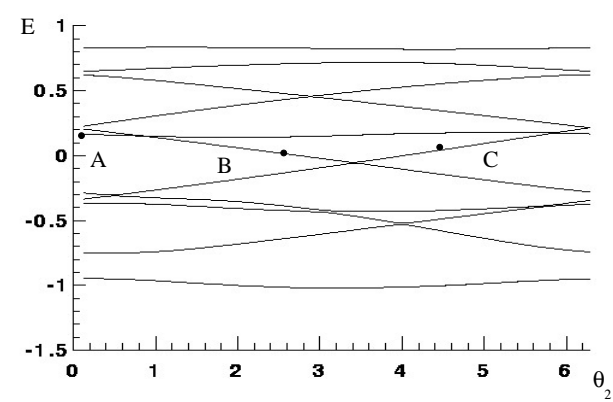

Figure 9. Energy levels $E_{n}\left(\theta_{1}, \theta_{2}\right)$ for $n=1 \rightarrow 11$, of Hamiltonian equation (3), as a function of $\theta_{2}$. There is no degeneracy, but the small splittings are not visible. Points $\mathrm{A}, \mathrm{B}$ and $\mathrm{C}$ correspond to the Husimi representations of figure 11 .

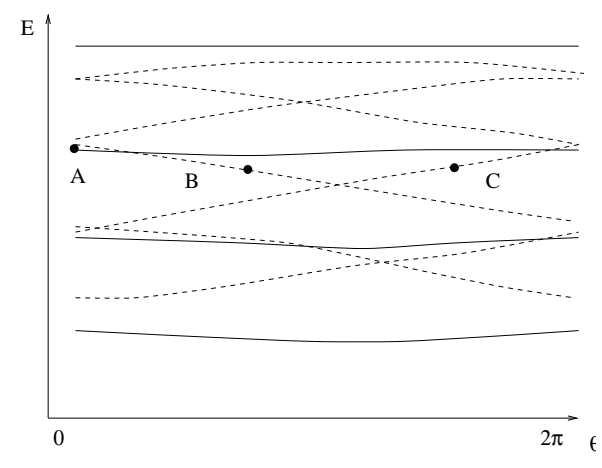

Figure 10. The semiclassical spectrum $\sigma_{s c}\left(\theta_{2}\right)$ obtained from figure (9). The four solid curves correspond to quasi-modes $\left|\tilde{\psi}_{c}\left(\theta_{2}\right)\right\rangle$ localized on contractible trajectories. The dashed curves correspond to quasi-modes $\left|\tilde{\psi}_{n c}\left(\theta_{2}\right)\right\rangle$ localized on noncontractible trajectories.
(B)

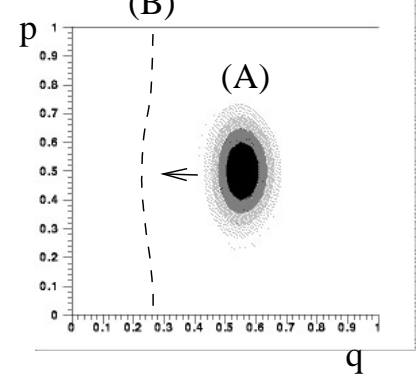

(B)

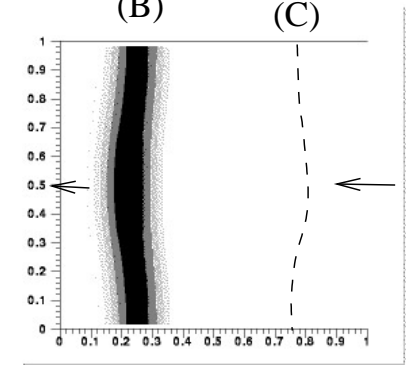

(C)

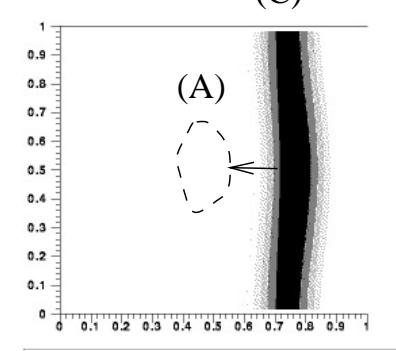

Figure 11. The dark density are the Husimi representation of eigenstates $\left|\varphi_{n}\left(\theta_{1}, \theta_{2}\right)\right\rangle$ for the band $n=6$ (see figure 9), with $\theta_{1}=0$, and three different values of $\theta_{2}$. (A): $\theta_{2}=0$; (B): $\theta_{2}=2,5$, (C): $\theta_{2}=4,5$. The dashed curves are the trajectories where the quasi-modes will jump at the next crossing.

Figure 11 shows the Husimi representation (see appendix A) of eigenstates $\left|\varphi_{n}\left(\theta_{1}, \theta_{2}\right)\right\rangle$ for the level $n=6$, with $\theta_{1}=0$, and three different values of $\theta_{2}$. These three eigenstates correspond to the points $\mathrm{A}-\mathrm{C}$ in figures 9 and 10. Looking at the classical trajectories in figure 1, one can clearly associate these quasi-modes respectively with the trajectories:

(A) With a contractible trajectory (point D on figure 1 ),

(B) A noncontractible trajectory of type $(0,+1)$,

(C) A noncontractible trajectory of type $(0,-1)$.

As $\theta_{2}$ varies from 0 to $2 \pi$, the quantum state $\left|\varphi_{6}\left(\theta_{1}, \theta_{2}\right)\right\rangle$ of level $n=6$ jumps. From figures 9 and 10 it is evident that there are three (avoided) level crossings, where the quasi-mode jumps.

Figure 11 shows that for the first crossing from A to B the quasi-mode stays in the same cell $\left(\Delta n_{1}=0\right)$. For the second crossing from $\mathrm{B}$ to $\mathrm{C}$ the quasi-mode changes by $\Delta n_{1}=-1$ cell. For the third crossing from $\mathrm{C}$ to $\mathrm{A}$, the quasi-mode stays in the same cell $\left(\Delta n_{1}=0\right)$. This gives a total change of $\Delta n_{1}=-1$ cell and a homotopy $I_{6}=-1$ for this sequence of 

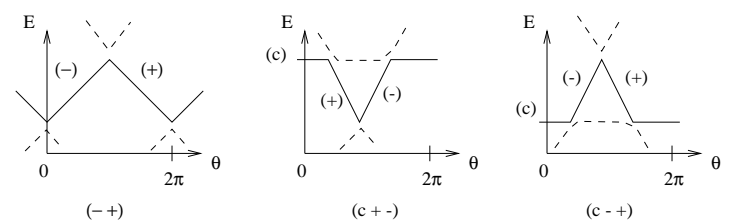

Figure 12. The three possible band structures (solid lines), made with a noncontractible trajectory of type $(0, \pm 1)$ and a contractible trajectory $(c)$. The dashed lines are the upper and lower bands.

quasi-modes. Accordingly, we have $C_{6}=I_{6}=-1$. Our result, equation (22), is well verified here.

\section{The Chern indices of the spectrum in a generic situation}

From the above results, we give here the precise values of Chern indices for a simple and generic Hamiltonian (i.e. stable under perturbations) corresponding to the numerical example equation (3) and explain how to read them from the Reeb graph (figure 2).

To simplify the discussion, we suppose in this section that we deal with a Hamiltonian $H$ such that the curve representing the noncontractible trajectories in the Reeb graph, has only one maximum and minimum, like the dashed curve in figure 2 . We suppose, moreover, that in the energy range of this curve, there is only one family of contractible trajectories, like the solid curve $\mathrm{CD}$ in figure 2.

In the range of energy of the noncontractible trajectories, each band of the spectrum is made with one of the three following sequences of quasi-modes, see figure 12:

- The band is made with the two noncontractible trajectories of type $(0, \pm 1)$. This gives the sequence: $S=(+-)$.

- The band has also a quasi-mode on the contractible trajectory $(c)$. This gives two possible sequences: $S=(c+-)$ or $S=(c-+)$.

To simplify the discussion, we exclude sequences as $(c+c-)$ or $(c-c+)$. (These sequences do not appear if the energy intervals between successive energy levels of contractible quasimodes are large enough.)

It is clear from figure 12 that if the band $n$ has the sequence $S_{n}=(c+-)$, then the band $n+1$ necessarily has the sequence $S_{n+1}=(c-+)$, and vice versa. These sequences always come in pairs.

To illustrate this from our numerical example, we can see in figures 9 and 10 that the bands 3-9 have, respectively, the sequences: $S_{3}=(c+-), S_{4}=(c-+), S_{5}=(+-)$, $S_{6}=(c+-), S_{7}=(c-+), S_{8}=(+-), S_{9}=(+-)$.

Now we saw in proposition (3) that a nonzero Chern index results from the jumping of neighbouring quasi-modes on the cylinder, via noncontractible trajectories. We also saw that a given quasi-mode $|\tilde{\psi}\rangle$ jumps by tunnelling effect to the nearest quasi-mode of the same energy located on a noncontractible trajectory. It is therefore important to determine in which direction (right or left) this jump occurs: we have to complete the Reeb graph (figure 2), by giving the direction of the shortest jump between any two trajectories. For that purpose, it is sufficient to give the location of the separatrix between left and right jumps.

In figure 13, we have reproduced the Reeb graph (the solid curve), and we have drawn (dashed curve and dotted curve) the possible location of this separatrix. The effect of this separatrix (dashed curve) is shown with the plot of the jumps from one particular quasi-mode $|\tilde{\psi}\rangle$ to a noncontractible trajectory of type (-1) at two different energies, on both sides of the separatrix. The separatrix for the jump from/to a noncontractible of type $(+1)$ is drawn with a dotted curve. 


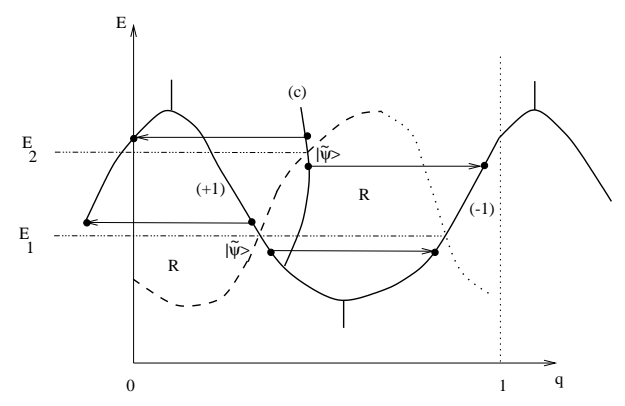

Figure 13. Example for the jumping of a quasi-mode $|\tilde{\psi}\rangle$ to the nearest noncontractible trajectory (of type $(0,-1)$ ). The solid curve is the Reeb graph as in figure 2. The dotted and dashed curves represent the separatrix for these attraction basins.

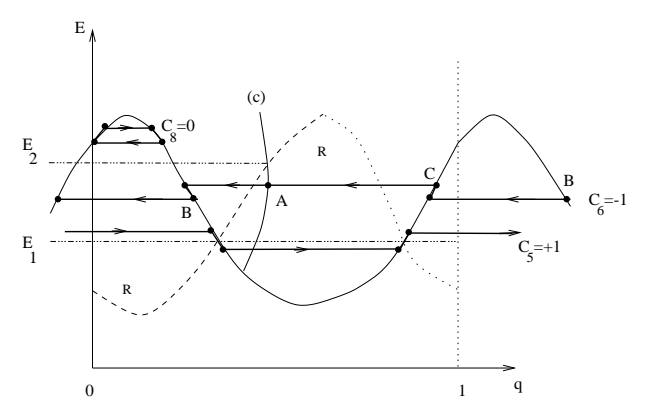

Figure 14. From knowledge of the sequences $S_{5}, S_{6}, S_{8}$, their homotopy and the Chern indices $C_{5}=+1, C_{6}=-1$ and $C_{8}=0$ are graphically computed. $\quad C_{5}=+1$ is associated with a band of energy $E_{1}$. Note that $C_{6}=-1$ is nonzero thanks to the quasi-mode (A) on the contractible trajectory (c) in region $R$.

$E_{1}$ is the energy where this separatrix crosses the noncontractible trajectory family. That the energy $E_{1}$ is the same for the dashed and dotted curves comes simply from the reciprocity property of the tunnelling 'geodesic'.

What matters, in fact, is only the intersection of this separatrix with the Reeb graph. Figure 13 is qualitative here, but quantitative calculations of the tunnelling effect would precisely determine the energy of these intersections.

Now, by combining figures 12 and 13 together with rule (22), it is easy to compute the Chern index of the three kind of bands. This is done in figure 14 for the sequences $S_{5}, S_{6}, S_{8}$, and we infer the general result:

- If $E_{1}$ is in the range of a band of type (+-) then its Chern index is $C_{(+-)}=+1$, otherwise $C_{(+-)}=0$.

- If the trajectory $(c)$ is in the region $R$ (of figure 13) then $C_{(c+-)}=-1$ (resp. +1 ), if the band has energy greater than $E_{1}$ (resp. lower). In the other regions, $C_{(c+-)}=0$.

- If the trajectory $(c)$ is in the region $R$ then $C_{(c-+)}=+1$ (resp. -1 ), if the band has energy greater than $E_{1}$ (resp. lower). In the other regions, $C_{(c-+)}=0$.

This allows us to interpret the Chern indices of our numerical example: the band $S_{5}=(+-)$ with $C_{5}=+1$ corresponds to the energy $E_{1}$. Above it, the two bands $S_{6}, S_{7}$ with Chern indices $C_{6}=-1, C_{7}=+1$ form a pair, thanks to the presence of the contractible trajectory $(c)$ in region $R$.

As a general result, we conclude that for any choice of Hamiltonian $H$ with a similar Reeb graph, there is a unique band in the middle of the spectrum, including energy $E_{1}$, with Chern index +1 . Above this energy (for $E_{1}<E<E_{2}$ ), the Chern indices come in pairs $(-1,+1)$, and under the energy $E_{1}$, the Chern indices come in pairs $(+1,-1)$.

Of course, for another appearance of the Reeb graph, the rules could be different. In particular, the Chern indices can reach higher values if the noncontractible curve of the Reeb graph has more than one maximum. We do not know if there a simple and explicit expression of the Chern indices in the general case.

\section{Chern index and Hall conductivity}

It is well known [1] that the Chern index $C$ of a band is related to the transverse integer quantum Hall conductivity $\sigma_{x y}$ by the relation equation (1). The usual demonstration of this formula 
starts from the Kubo linear response formula for $\sigma_{x y}$, and expresses it as the curvature integral for $C$, equation (20). In this section we want to demonstrate it from another point of view, by relating $C$ with the motion of a wavepacket on the plane $T_{q p}$. This result seems more intuitive.

Consider a given energy band $n$ of the spectrum equation (11). For each $\left(\theta_{1}, \theta_{2}\right)$ there is one eigenvector $\left|\varphi_{n}\left(\theta_{1}, \theta_{2}\right)\right\rangle$ defined up to a multiplicative constant. Suppose that we have a $C^{\infty}$ function $\left(\theta_{1}, \theta_{2}\right) \rightarrow\left|\psi\left(\theta_{1}, \theta_{2}\right)\right\rangle \in \mathcal{H}_{T}\left(\theta_{1}, \theta_{2}\right)$, where $\left|\psi\left(\theta_{1}, \theta_{2}\right)\right\rangle$ is proportional to $\left|\varphi_{n}\left(\theta_{1}, \theta_{2}\right)\right\rangle$, equation (11). Note that if $C_{n} \neq 0$, this function must be zero at some values of $\left(\theta_{1}, \theta_{2}\right)$.

Consider now

$$
|\psi\rangle=\iint \mathrm{d} \theta_{1} \mathrm{~d} \theta_{2}\left|\psi\left(\theta_{1}, \theta_{2}\right)\right\rangle
$$

Then $|\psi\rangle \in \mathcal{H}_{P}=L^{2}(\mathbb{R})$. We can say that $|\psi\rangle$ is a state 'localized' on the plane $T_{q p}$ constructed from the band $n$, like Wannier states in solid state physics. (Conversely, given $|\Phi\rangle \in L^{2}(\mathbb{R})$, we can project it on the spectral band $n$ and obtain such $|\psi\rangle$.)

The state $|\psi\rangle$ can be translated by $\left(n_{1}, n_{2}\right)$ cells on the plane by

$$
\left|\psi_{n_{1}, n_{2}}\right\rangle=T_{Q}^{n_{1}} T_{P}^{n_{2}}|\psi\rangle=\iint \mathrm{d} \theta_{1} \mathrm{~d} \theta_{2} \mathrm{e}^{\mathrm{i} n_{1} \theta_{1}+\mathrm{i} n_{2} \theta_{2}}\left|\psi\left(\theta_{1}, \theta_{2}\right)\right\rangle .
$$

Conversely,

$$
\left|\psi\left(\theta_{1}, \theta_{2}\right)\right\rangle=\sum_{n_{1}, n_{2} \in \mathbb{Z}^{2}} \mathrm{e}^{-\mathrm{i} n_{1} \theta_{1}-\mathrm{i} n_{2} \theta_{2}}\left|\psi_{n_{1}, n_{2}}\right\rangle .
$$

These simple relations can be generalized by the following result.

Consider another state constructed from the band $n$ :

$$
|\phi\rangle=\iint \mathrm{d} \theta_{1} \mathrm{~d} \theta_{2} \mathrm{e}^{\mathrm{i} f\left(\theta_{1}, \theta_{2}\right)}\left|\psi\left(\theta_{1}, \theta_{2}\right)\right\rangle
$$

where $f$ is a continuous and periodic function such that

$$
\begin{aligned}
& f\left(\theta_{1}+2 \pi, \theta_{2}\right)=f\left(\theta_{1}, \theta_{2}\right)+N_{1} 2 \pi \\
& f\left(\theta_{1}, \theta_{2}+2 \pi\right)=f\left(\theta_{1}, \theta_{2}\right)+N_{2} 2 \pi
\end{aligned}
$$

$\left(N_{1}, N_{2} \in \mathbb{Z}^{2}\right.$ are topological integers which characterized the homotopy of the function $f$ ).

Let us define

$$
\begin{aligned}
& \left\langle n_{1}\right\rangle=\sum_{n_{1}, n_{2}} n_{1} \cdot\left|\left\langle\psi_{n_{1}, n_{2}} \mid \phi\right\rangle\right|^{2} \\
& \left\langle n_{2}\right\rangle=\sum_{n_{1}, n_{2}} n_{2} \cdot\left|\left\langle\psi_{n_{1}, n_{2}} \mid \phi\right\rangle\right|^{2}
\end{aligned}
$$

which give the mean position of the state $|\phi\rangle$ on the plane $T_{q p}$ relatively to the localized states $\left|\psi_{n_{1}, n_{2}}\right\rangle$. It is easy to prove that

$$
\begin{aligned}
& \left\langle n_{1}\right\rangle=N_{1} \\
& \left\langle n_{2}\right\rangle=N_{2} .
\end{aligned}
$$

The mean position of $|\phi\rangle$ is then 'quantized'.

(This is a simple property of Fourier series: if $g(\theta)=\sum_{n} c_{n} \mathrm{e}^{\mathrm{i} n \theta}=\mathrm{e}^{\mathrm{i} f(\theta)}$ is periodic and $f(2 \pi)=f(0)+N 2 \pi$ then $N=\sum_{n} n\left|c_{n}\right|^{2}$. Another way to say it is: $\sum_{n} n\left|c_{n}\right|^{2}=$ $\left\langle g\left|\hat{p}_{\theta}\right| g\right\rangle=\frac{1}{2 \pi} \int_{0}^{2 \pi} f^{\prime} \mathrm{d} \theta=N$, with the current operator $\hat{p}_{\theta}=\frac{1}{\mathrm{i}} \mathrm{d} / \mathrm{d} \theta$.)

We now give some consequences of this result on the 'quantized mean position'.

Now let us first look at the time evolution of $\left|\psi_{0,0}\right\rangle$ :

$$
|\psi(t)\rangle=\mathrm{e}^{-\mathrm{i} H t / \hbar}\left|\psi_{0,0}\right\rangle=\iint \mathrm{d} \theta_{1} \mathrm{~d} \theta_{2} \mathrm{e}^{-\mathrm{i} E\left(\theta_{1}, \theta_{2}\right) t / \hbar}\left|\psi\left(\theta_{1}, \theta_{2}\right)\right\rangle .
$$


In comparison with equation (23), the phase function is the dynamical phase $f\left(\theta_{1}, \theta_{2}\right)=$ $-E\left(\theta_{1}, \theta_{2}\right) t / \hbar$ of homotopy type $N_{1}=N_{2}=0$ (because by continuous homotopy $t \rightarrow 0, f$ is mapped to 0 ). This means that during its evolution, the quantum state $|\psi(t)\rangle$ spreads over the plane, but its mean position $\left\langle n_{1}\right\rangle,\left\langle n_{2}\right\rangle$ stays zero.

Let us suppose now that $\theta_{1}, \theta_{2}$ are no more 'good quantum numbers', and that there is a slow drift. In the model of bi-dimensional electrons of the introduction, this drift is the adiabatic motion due to the low electrical field $E_{x}$. Consider, for example,

$$
\begin{aligned}
& \theta_{1}(t)=-\omega_{1} t \\
& \theta_{2}(t)=\theta_{2}(0) .
\end{aligned}
$$

After one period $T=2 \pi / \omega_{1}$, the evolution gives

$$
\mathrm{e}^{-\mathrm{i} H T / \hbar}\left|\psi\left(\theta_{1}, \theta_{2}\right)\right\rangle=\exp \left(\mathrm{i} \phi_{D}\left(\theta_{1}, \theta_{2}\right)+\mathrm{i} \phi_{B}\left(\theta_{2}\right)\right)\left|\psi\left(\theta_{1}, \theta_{2}\right)\right\rangle
$$

where $\phi_{D}\left(\theta_{1}, \theta_{2}\right)$ is the dynamical phase of homotopy type $N_{1}=N_{2}=0$ and $\phi_{B}\left(\theta_{1}, \theta_{2}\right)$ is the Berry phase of the path $\theta_{1}(t)$ and does not depend on $\theta_{1}$ henceforth. By homotopy deformation, $\phi_{B}\left(\theta_{2}\right) \equiv 2 \pi C \theta_{2}$ and is therefore of type $N_{1}=0, N_{2}=C$. This means that after one period $T$, the mean position of the quantum state $|\psi(t)\rangle$ has been changed by $\delta\left\langle n_{1}\right\rangle=0, \delta\left\langle n_{2}\right\rangle=C$ (in cells units).

This 'quantized' velocity $V_{2}=\delta\left\langle n_{2}\right\rangle / T$ is responsible for the integer Hall conductivity. Indeed, in the Harper model [1] of noninteracting bi-dimensional $(x, y)$ electrons in a biperiodic potential of period $(X, Y)$, and a perpendicular high magnetic field $B_{z}$, with a low electrical field along $E_{x}$, the adiabatic hypothesis gives

$$
\theta_{1}(t)=-\frac{e X}{\hbar} E_{x} t
$$

We have obtained that the quantized velocity of one-electron quantum state is $V_{y}=(C Y) / T$. For a filled band, the electronic density is one electron per cell: $\rho=1 /(X Y)$. The electronic density current of a filled band is then $j_{y}=\rho e V_{y}=\frac{e^{2}}{h} C \cdot E_{x}$ which leads to equation (1).

\section{Conclusion}

By using quasi-modes, we have obtained a semiclassical description of band eigenstates of a generic quantum Hamiltonian on the torus phase space. By using general results exposed in the appendices, relating the topological Chern index of a band, with the localization properties of the quantum states, we have been able to express the Chern index of each band in terms of the motion of the quasi-modes on the classical phase space.

In section 7, we have shown how the Chern indices of the whole spectrum can be graphically computed from the classical Reeb graph. There is an interesting question which can be raised from our work: conversely, from a given sequence $\left(C_{n}\right)_{n}$ of integers such that $\sum_{n} C_{n}=+1$, the question would be to determine the potential $V(x, y)$ whose band spectrum possesses this precise sequence of Chern indices.

Currently, one tries to observe experimental signatures of the Harper spectrum (Landau level substructures) in superlattices with periods of about $100 \mathrm{~nm}$ on GA-As-AlGaAs heterojunctions $[27,28]$. In these experiments, the Chern index gives the quantum Hall conductivity $\sigma_{x y}$. In this paper we have shown simple correspondances between the Chern index and the classical trajectories of the electrons. These correspondances could be observed in experiments by measuring the variations of the integer Hall conductivity, in relation with the potential $V(x, y)$ of the supperlattice, which may be created by an external electrostatic grid. 


\section{Acknowledgments}

Discussions with B Parisse and Y Colin de Verdiere are gratefully acknowledged.

\section{Appendix A. Bargmann and Husimi representation}

In this appendix, we recall well known results on Bargmann and Husimi representation on the torus phase space [19].

We have seen previously that the space $\mathcal{H}_{T}$ is not a subspace of $L^{2}(\mathbb{R})$. For functions belonging to $\mathcal{H}_{T}$, it will be more useful to introduce a phase space representation of the quantum states, called the Bargmann representation [29].

Consider a quantum state $|\psi\rangle \in L^{2}(\mathbb{R})$. In order to characterize the localization of $|\psi\rangle$ in the phase space near the point $(q, p)$, we first construct a Gaussian wavepacket $|q p\rangle$ (coherent state) defined in the $x$-representation by

$$
\langle x \mid q p\rangle=\left(\frac{1}{\pi \hbar}\right)^{1 / 4} \exp \left(\frac{\mathrm{i}}{\hbar} p x\right) \exp \left(-\frac{(x-q)^{2}}{2 \hbar}\right) .
$$

The notation $|q p\rangle$ recalls that the coherent state is localized (in the semiclassical limit) at the point $(q, p)$ of the phase space.

The Husimi distribution of a state $|\psi\rangle$ is defined over the phase space by

$$
h_{\psi}(q, p)=|\langle q p \mid \psi\rangle|^{2}
$$

and for $\varphi \in L^{2}(\mathbb{R})$, we have

$$
\int|\varphi(x)|^{2} \mathrm{~d} x=\iint|\langle q p \mid \varphi\rangle|^{2} \frac{\mathrm{d} q \mathrm{~d} p}{2 \pi \hbar} .
$$

To characterize the functions of $L^{2}\left(T^{*} \mathbb{R}\right)$ which are $(q, p)$ representations of a state, it is more convenient to introduce a complex-representation of the phase space $z=\frac{1}{\sqrt{2 \hbar}}(q+\mathrm{i} p)$. Another (proportional) expression of the coherent state is then

$$
|z\rangle=\exp \left(z a^{+}\right)|0\rangle
$$

with $|0\rangle$ being the fundamental of the harmonic oscillator $H_{0}=\hat{q}^{2}+\hat{p}^{2}$, and $a^{+}$being the associated creation operator. Indeed:

$$
|q p\rangle=\exp \left(\mathrm{i} \frac{q p}{2 \hbar}-\frac{q^{2}+p^{2}}{4 \hbar}\right)|z\rangle
$$

The following anti-holomorphic function of $z$ is called the Bargmann distribution of $\psi$ :

$$
b_{\psi}(z)=\langle z \mid \psi\rangle \text {. }
$$

Clearly, we have

$$
h_{\psi}(q, p)=\left|b_{\psi}(z)\right|^{2} \mathrm{e}^{-\frac{q^{2}+p^{2}}{2 h}}
$$

hence the zeros of the function $h_{\psi}(q, p)$ are those of the holomorphic function $b_{\psi}(z)$, which are localized zeros in the phase space. Moreover, (24) implies that $\psi \in L^{2}(\mathbb{R})$ if and only if $b_{\psi} \in L^{2}\left(\mathbb{C}, \mathrm{e}^{-|z|^{2} / h}\right)$ and $b_{\psi}$ is anti-holomorphic.

The same definitions can be applied for a state $|\psi\rangle \in \mathcal{H}_{T}\left(\theta_{1}, \theta_{2}\right)$. The corresponding Bargmann function is a theta-function [30] and the Husimi distribution is bi-periodic in $(q, p)$, hence is well defined on the torus $T_{q p}$.

We need the following properties concerning the Bargmann representation $b_{\psi}(z)=\langle z \mid \psi\rangle$ of a quantum state $|\psi\rangle$ on the torus $(|z\rangle$ is a coherent state on the torus [31]. 
If $|\psi\rangle \in \mathcal{H}_{T}\left(\theta_{1}, \theta_{2}\right)$, the Bargmann function $b_{\psi}(z)$ is (a theta-function) anti-holomorphic with respect to $z$, and with $N$ zeros $Z=\left(z_{1}, \ldots, z_{N}\right)$ in the cell $[0,1] \times[0,1]$. These zeros are constrained by ([30], this is the 'Abel theorem' and 'Jacobi inversion theorem', see [15] p 235):

$$
\begin{aligned}
& \sum_{i=1}^{N} q_{i}=\frac{\theta_{2}}{2 \pi} \\
& \sum_{i=1}^{N} p_{i}=\frac{\theta_{1}}{2 \pi}
\end{aligned}
$$

with $z_{i}=q_{i}+\mathrm{i} p_{i}$. Conversely, such a collection of $N$ zeros define a state $|\psi\rangle \in \mathcal{H}_{T}\left(\theta_{1}, \theta_{2}\right)$, unique up to a multiplicative constant.

Note that since the $N$ zeros are constrained to have a fixed sum, we get the right dimension $N-1+1$ for the Hilbert space $\mathcal{H}_{T}\left(\theta_{1}, \theta_{2}\right)$.

\section{Appendix B. Calculation of the Chern index in special cases}

For simplicity, we will note $\theta=\left(\theta_{1}, \theta_{2}\right) \in \mathbb{R}^{2}$.

We will compute the Chern index of a line bundle in particular cases. We will always consider a line sub-bundle of $\mathcal{H}_{T}(\theta) \rightarrow T_{\theta}$. Such a line bundle $F$ is characterized by giving local section $\theta \rightarrow\left|\psi_{i}(\theta)\right\rangle \in \mathcal{H}_{T}(\theta)$ over open sets $U_{i}$ which cover $T_{\theta}$.

From appendix A, the line bundle $F$ is also characterized by the function $\theta \rightarrow Z(\theta)$, where $Z(\theta)$ is the set of $N$ nonordered zeros of the Bargmann functions $b_{\psi(\theta)}(z)$.

\section{B.1. Theorem on homotopy invariance}

Theorem 1. Consider two complex line bundles over $T_{\theta}$ with Chern index $C$ and $C^{\prime}$ defined by local sections $\left|\psi_{i}(\theta)\right\rangle$ and $\left|\psi_{i}^{\prime}(\theta)\right\rangle$ on open sets $U_{i}$. Suppose, moreover, that

$$
\left\langle\psi_{i}(\theta) \mid \psi_{i}^{\prime}(\theta)\right\rangle \neq 0 \quad \forall \theta \quad \forall i
$$

then $C=C^{\prime}$.

Proof. We can suppose that the sections are normalized $\left(\left\langle\psi_{i}(\theta) \mid \psi_{i}(\theta)\right\rangle=1\right)$. On a set $U_{i}$, and for a given $\theta$, define

$$
\rho_{i}(\theta)=\left|\left\langle\psi_{i}(\theta) \mid \psi_{i}^{\prime}(\theta)\right\rangle\right| \leqslant 1
$$

and for fixed $\lambda \in[0,1]$ :

$$
\left|\varphi_{i, \lambda}(\theta)\right\rangle=\left|\psi_{i}(\theta)\right\rangle \cdot\left\langle\psi_{i}(\theta) \mid \psi_{i}^{\prime}(\theta)\right\rangle+\lambda\left(\left|\psi_{i}^{\prime}(\theta)\right\rangle-\left|\psi_{i}(\theta)\right\rangle\left\langle\psi_{i}(\theta) \mid \psi_{i}^{\prime}(\theta)\right\rangle\right) .
$$

In the theorem, we suppose that $\left.\rho_{i}(\theta)\right\rangle 0$. Thus

$$
\left.\left\langle\psi_{i}^{\prime}(\theta) \mid \varphi_{i, \lambda}(\theta)\right\rangle=\rho^{2}+\lambda\left(1-\rho^{2}\right)\right\rangle 0
$$

and then $\left|\varphi_{i, \lambda}(\theta)\right\rangle \neq 0$.

We now check that the sections $\left|\varphi_{i, \lambda}(\theta)\right\rangle$ define a complex line bundle $F_{\lambda}$. On another set $U_{j}$, if $\left|\psi_{j}(\theta)\right\rangle=\exp (\mathrm{i} \alpha)\left|\psi_{i}(\theta)\right\rangle$ and $\left|\psi_{j}^{\prime}(\theta)\right\rangle=\exp (\mathrm{i} \beta)\left|\psi_{i}^{\prime}(\theta)\right\rangle$ then $\left|\varphi_{j, \lambda}(\theta)\right\rangle=$ $\exp (\mathrm{i} \beta)\left|\varphi_{i, \lambda}(\theta)\right\rangle$ is in the same line as $\left|\varphi_{i, \lambda}(\theta)\right\rangle$.

We have thus obtain a homotopic deformation $F_{\lambda}, \lambda \in[0,1]$ between the line bundle $F_{0}$ and $F_{1}$. They have thus the same Chern index $C=C_{\lambda}=C^{\prime}$. 
Theorem 2. More generally ([15]p 141) if we suppose that

$$
\theta \rightarrow\left\langle\psi_{i}(\theta) \mid \psi_{i}^{\prime}(\theta)\right\rangle
$$

has zeros in $\theta^{*}$ with index $\iota\left(\theta^{*}\right)= \pm 1$, then

$$
C=C^{\prime}+\sum_{\operatorname{zeros} \theta^{*}} \iota\left(\theta^{*}\right) \text {. }
$$

\section{B.2. The Chern index from the zeros of the Bargmann function}

Let $F$ be a line bundle as above.

- Suppose that there exists some point $z_{0} \in T_{q p}$ of phase space, such that

$$
\forall \theta \in T_{\theta} \quad b_{\psi(\theta)}\left(z_{0}\right) \neq 0
$$

(equivalently, this means that $z_{0} \notin Z(\theta)$, for all $\theta$ ). Then we can select a vector $|\psi(\theta)\rangle \in H_{N}(\theta)$ in each fibre such that $\arg \left(b_{\psi(\theta)}\left(z_{0}\right)\right)=0$. This gives a nonvanishing global section of the bundle. This is also a global frame, hence the bundle is trivial, and $C=0$.

- More generally, define

$$
N\left(z_{0}\right)=\left\{\theta \in T_{\theta} / z_{0} \in Z(\theta)\right\}
$$

then

$$
C=\sum_{\theta \in N\left(z_{0}\right)}( \pm 1)
$$

where the sign \pm 1 corresponds to the local orientation of the mapping $z_{i}$ at $\theta$, where $Z=\left\{z_{1}, \ldots, z_{N}\right\}$ and $z_{i}(\theta)=z_{0}$. This can be deduced directly from equation (26), with $\left|\psi^{\prime}\right\rangle=\left|z_{0}\right\rangle$.

\section{B.3. Example of a line bundle with a given Chern index $C$}

We now construct explicit examples of such a bundle.

- Suppose that $N \geqslant 2$. We define a line bundle $F_{0}$, by specifying the zeros of the Bargmann representation of a section, with the notation of equation (25):

$$
\begin{aligned}
& q_{1}=\frac{\theta_{2}}{2 \pi} \quad p_{1}=0 \\
& q_{2}=0 \quad p_{2}=\frac{\theta_{1}}{2 \pi} \\
& q_{i}=0 \quad p_{i}=0 \quad \text { for } \quad 3 \leqslant i \leqslant N .
\end{aligned}
$$

The constraint equation (25) is easily verified. If we choose $z_{0}=\left(q_{0}+\mathrm{i} p_{0}\right)$ with $q_{0}=p_{0}=0.5, z_{0} \notin Z(\theta)$ and from equation (27), we deduce that the bundle $F_{0}$ is trivial, with Chern index $C=0$.

- More generally, consider the bundle $F_{C}$ defined by

$$
\begin{aligned}
& q_{1}=(1-C) \frac{\theta_{1}}{2 \pi} \quad p_{1}=0 \\
& q_{2}=C \frac{\theta_{2}}{2 \pi} \quad p_{2}=\frac{\theta_{1}}{2 \pi} \\
& q_{i}=0 \quad p_{i}=0 \quad \text { for } \quad 3 \leqslant i \leqslant N .
\end{aligned}
$$

The constraint (25) is verified. We can apply equation (27), to calculate the Chern index $C$. The set $N\left(z_{0}\right)$ is given by

and we obtain that the Chern index is $C$.

$$
\theta_{1}=\pi[2 \pi] \quad \theta_{2}=\frac{\pi}{C}[2 \pi / C]
$$


B.4. Chern index for a moving coherent state

Theorem 3. Suppose $N \geqslant 2$. Consider the line bundle $F_{z}$ defined by the local section $|\psi(\theta)\rangle=\left|z_{\theta}\right\rangle \in \mathcal{H}_{T}(\theta)$ with $z_{\theta}=q_{\theta}+\mathrm{i} p_{\theta}$, and

$$
\left(\begin{array}{c}
q_{\theta} \\
p_{\theta}
\end{array}\right)=\left(\begin{array}{ll}
n_{11} & n_{12} \\
n_{21} & n_{22}
\end{array}\right)\left(\begin{array}{l}
\theta_{1} / 2 \pi \\
\theta_{2} / 2 \pi
\end{array}\right)
$$

(This means that the coherent state $\left|z_{\theta}\right\rangle$ is moving over the cells as $\theta$ is varying.)

The Chern index of this bundle is then

$$
C=N \cdot\left|\begin{array}{ll}
n_{11} & n_{12} \\
n_{21} & n_{22}
\end{array}\right|+n_{21}+n_{12} .
$$

Proof. Consider the line bundle $F_{0}$ defined by the zeros equation (28), with local section noted $\left|\varphi_{i}(\theta)\right\rangle$ on each open set $U_{i} \subset T_{\theta}$. This bundle has $C=0$ Chern index. Consider the function

$$
f: \theta \rightarrow\left\langle\varphi_{i}(\theta) \mid z_{\theta}\right\rangle .
$$

From equation (26), the Chern index $C$ of the bundle $F_{z}$ is

$$
C=\sum_{\theta^{*} \text { zeros of } f} \iota\left(\theta^{*}\right) .
$$

The zeros of $f$ are given by

$\left\langle\varphi_{i}(\theta) \mid z_{\theta}\right\rangle=0 \Leftrightarrow z_{\theta}$ is a zero of the section $\left|\varphi_{i}(\theta)\right\rangle$

$$
\Leftrightarrow\left\{\begin{array}{l}
q_{\theta}=q_{i} \\
p_{\theta}=p_{i}
\end{array} \quad i=1, \ldots, N\right.
$$

this gives

$$
\begin{aligned}
&\left\{\begin{array}{l}
n_{11} \frac{\theta_{1}}{2 \pi}+n_{12} \frac{\theta_{2}}{2 \pi} \\
n_{21} \frac{\theta_{1}}{2 \pi}+n_{22} \frac{\theta_{2}}{2 \pi}
\end{array}=\left\{\begin{array}{l}
\frac{\theta_{2}}{2 \pi} \\
0
\end{array} \quad \text { or } \quad=\left\{\begin{array}{l}
0 \\
\frac{\theta_{1}}{2 \pi}
\end{array}\right. \text { or }\right.\right. \\
&=\left\{\begin{array}{l}
0 \\
0
\end{array} \text { for } \quad i=3, \ldots, N .\right.
\end{aligned}
$$

We deduce from equation (2) that

$$
\begin{gathered}
C=\left|\begin{array}{cc}
n_{11} & n_{12}-1 \\
n_{21} & n_{22}
\end{array}\right|+\left|\begin{array}{cc}
n_{11} & n_{12} \\
n_{21}-1 & n_{22}
\end{array}\right|+(N-2)\left|\begin{array}{ll}
n_{11} & n_{12} \\
n_{21} & n_{22}
\end{array}\right| \\
=N\left|\begin{array}{ll}
n_{11} & n_{12} \\
n_{21} & n_{22}
\end{array}\right|+n_{21}+n_{12} .
\end{gathered}
$$

\section{B.5. Bundle constructed from periodic motion of states on the plane}

A natural question is: is it possible to generalize theorem 3, for the periodic motion of arbitrary states in $L^{2}(\mathbb{R})$ rather than only coherent states?

There is a first result, theorem 4 , as follows. 
Theorem 4. Let $S: \theta \rightarrow|\psi(\theta)\rangle \in L^{2}(\mathbb{R})$ for $\theta \in \mathbb{R}^{2}$ be an arbitrary mapping such that

$$
\begin{aligned}
& \forall \theta \quad|\psi(\theta)\rangle \neq 0 \\
& \left|\psi\left(\theta_{1}+2 \pi, \theta_{2}\right)\right\rangle=T_{Q}^{n_{11}} T_{P}^{n_{12}}|\psi(\theta)\rangle \\
& \left|\psi\left(\theta_{1}, \theta_{2}+2 \pi\right)\right\rangle=T_{Q}^{n_{21}} T_{P}^{n_{22}}|\psi(\theta)\rangle
\end{aligned}
$$

with $\left(n_{11}, n_{12}, n_{21}, n_{22}\right) \in \mathbb{Z}^{4}$ which characterizes the periodic motion of the states $|\psi(\theta)\rangle$ as $\theta$ is varied.

For a contractible open subset $U$ of $T_{\theta}$, define

$$
|\tilde{\psi}(\theta)\rangle=P_{\theta_{1}} P_{\theta_{2}}(|\psi(\theta)\rangle) \in \mathcal{H}_{T}(\theta) .
$$

Then if $N \geqslant 3$, and for a generic map $S,|\tilde{\psi}(\theta)\rangle$ gives a local section of a well defined line bundle over $T_{\theta}$, which can be also noted $S$.

Proof. For every $\theta$, the line bundle is defined by the vector $|\tilde{\psi}(\theta)\rangle$. We have therefore to check that $|\tilde{\psi}(\theta)\rangle \neq 0$, and that this line is periodic with respect to $\theta$.

Because the space $\mathcal{H}_{T}(\theta)$ is $N$-dimensional, the condition $|\tilde{\psi}(\theta)\rangle=0$ is $N$-dimensional. As soon as $N>2$, and for $\theta \in[0,2 \pi]^{2}$ the condition $|\tilde{\psi}(\theta)\rangle=0$ cannot be satisfied generically. Now

$$
\begin{aligned}
\left|\tilde{\psi}\left(\theta_{1}+2 \pi, \theta_{2}\right)\right\rangle & =P_{\theta_{1}+2 \pi}\left(\left|\psi\left(\theta_{1}+2 \pi\right)\right\rangle\right) \\
& =P_{\theta_{1}} T_{Q}^{n_{11}} T_{P}^{n_{12}}\left|\psi\left(\theta_{1}\right)\right\rangle \\
& =\exp \left(\operatorname{in}_{11} \theta_{1}\right) \exp \left(\operatorname{in}_{12} \theta_{2}\right)|\tilde{\psi}(\theta)\rangle .
\end{aligned}
$$

So $|\tilde{\psi}(\theta)\rangle$ and $\left|\tilde{\psi}\left(\theta_{1}+a 2 \pi, \theta_{2}+b 2 \pi\right)\right\rangle$ are proportional and define the same line $\forall(a, b) \in \mathbb{Z}^{2}$.

Theorem 5. Suppose that $N \geqslant 4$. Let $S: \theta \rightarrow|\psi(\theta)\rangle \in L^{2}(\mathbb{R})$ be a generic mapping as defined in theorem 4. Then the Chern index of the line bundle $S$ is given by formula (30).

Proof. We note $S_{0}=S$, and $S_{1}=F_{z}$ the line bundle defined in theorem 3. The proof consists in constructing a homotopic deformation $S_{\lambda}$ from $S_{0}$ to $S_{1}$ for $\lambda \in[0,1]$. Because the Chern index $C_{\lambda}$ of $S_{\lambda}$ is constant, we therefore conclude that the Chern index of $S$ is given by formula (30).

For $\lambda \in[0,1]$, and $\theta \in \mathbb{R}^{2}$, define

$$
\left|\psi_{\lambda}(\theta)\right\rangle=\lambda|\psi(\theta)\rangle+(1-\lambda)|z(\theta)\rangle \in L^{2}(\mathbb{R})
$$

where $|z(\theta)\rangle$ is a standard coherent state on the plane, $z(\theta)=q_{\theta}+\mathrm{i} p_{\theta}$, with $q_{\theta}, p_{\theta}$ given by equation (29). We suppose, moreover, that the phases are chosen such that equation (31) holds for $|z(\theta)\rangle$ and $|\psi(\theta)\rangle$.

Then $\left|\psi_{\lambda}(\theta)\right\rangle=0$ if and only if $\lambda=\frac{1}{2}$ and $|\psi(\theta)\rangle=-|z(\theta)\rangle$. This last situation is nongeneric, and if it occurs, we can just choose $z(\theta)=\left(q_{\theta}+q_{0}\right)+\mathrm{i}\left(p_{\theta}+p_{0}\right)$ with arbitrary $q_{0}, p_{0}$ to avoid this. So $S_{\lambda}: \theta \rightarrow\left|\psi_{\lambda}(\theta)\right\rangle \in L^{2}(\mathbb{R})$ fulfils theorem 4 , and defines a line bundle for every $\lambda$. Because there are three parameters $\theta_{1}, \theta_{2}, \lambda$ we have to suppose now that $N \geqslant 4$, so that $|\tilde{\psi}(\theta)\rangle \neq 0$. 


\section{B.6. Bundle constructed from a periodic motion on the cylinder}

In section 4, we obtain the periodic motion of quasi-modes on the cylinder $C_{q p}$ phase-space. A slightly different result than theorem 5 is then needed.

Theorem 6. Suppose $N \geqslant 4$, and let

$$
\theta_{2} \in \mathbb{R} \rightarrow\left|\psi_{C}\left(\theta_{2}\right)\right\rangle \in \mathcal{H}_{C}\left(\theta_{2}\right)
$$

be a generic continuous mapping such that

$$
\begin{aligned}
& \forall \theta_{2} \in \mathbb{R} \quad\left|\psi_{C}\left(\theta_{2}\right)\right\rangle \neq 0 \\
& \left|\psi_{C}\left(\theta_{2}+2 \pi\right)\right\rangle=T_{Q}^{I}\left|\psi_{C}\left(\theta_{2}\right)\right\rangle
\end{aligned}
$$

with $I \in \mathbb{Z}$.

For $\theta=\left(\theta_{1}, \theta_{2}\right)$ define

$$
|\tilde{\psi}(\theta)\rangle=P_{\theta_{1}}\left|\psi_{C}\left(\theta_{2}\right)\right\rangle \in \mathcal{H}_{T}(\theta) .
$$

Then $\theta \rightarrow|\tilde{\psi}(\theta)\rangle \in \mathcal{H}_{T}(\theta)$ is a local section of a well defined line bundle over $T_{\theta}$, with Chern index $I$

Proof. The proof is similar to that of theorem 5.

The bundle is well defined because, first $|\tilde{\psi}(\theta)\rangle=0$ needs $N$ conditions, generically not realized for arbitrary $\theta_{1}, \theta_{2} \in[0,2 \pi]$, as soon as $N>2$.

Secondly, $|\tilde{\psi}(\theta)\rangle$ and $\left|\tilde{\psi}\left(\theta_{1}+a 2 \pi, \theta_{2}+b 2 \pi\right)\right\rangle=P_{\theta_{1}} T_{Q}^{I b}\left|\psi_{C}\left(\theta_{2}\right)\right\rangle=\exp \left(\mathrm{i} I b \theta_{1}\right)|\tilde{\psi}(\theta)\rangle$ are proportional and define therefore the same line.

In order to calculate the Chern index, consider the mapping

$$
\theta_{2} \in \mathbb{R} \rightarrow\left|z\left(\theta_{2}\right)\right\rangle \in L^{2}(\mathbb{R})
$$

where $\left|z\left(\theta_{2}\right)\right\rangle$ is a standard coherent state on the plane, $z\left(\theta_{2}\right)=q_{\theta}+\mathrm{i} p_{\theta}$, and

$$
\begin{aligned}
& q_{\theta}=I \cdot \theta_{2} \\
& p_{\theta}=p_{0}=\text { constant } .
\end{aligned}
$$

We can therefore construct $\left|z\left(\theta_{2}\right)_{C}\right\rangle=P_{\theta_{2}}\left|z\left(\theta_{2}\right)\right\rangle \in \mathcal{H}_{C}\left(\theta_{2}\right)$ and $\left|z\left(\theta_{1}, \theta_{2}\right)\right\rangle=P_{\theta_{1}}\left|z\left(\theta_{2}\right)_{C}\right\rangle \in$ $\mathcal{H}_{T}(\theta)$.

From theorem 3, $\theta \rightarrow\left|z\left(\theta_{1}, \theta_{2}\right)\right\rangle$ define a line bundle with Chern index $n_{12}=I$.

Consider now for $\lambda \in[0,1]$

$$
\theta_{2} \rightarrow\left|\psi_{\lambda}\left(\theta_{2}\right)\right\rangle=\lambda\left|\psi\left(\theta_{2}\right)\right\rangle+(1-\lambda)\left|z\left(\theta_{2}\right)_{C}\right\rangle \in \mathcal{H}_{C}\left(\theta_{2}\right)
$$

and

$$
S_{\lambda}: \theta=\left(\theta_{1}, \theta_{2}\right) \rightarrow\left|\tilde{\psi}_{\lambda}(\theta)\right\rangle=P_{\theta_{1}}\left|\psi_{\lambda}\left(\theta_{2}\right)\right\rangle \in H_{T}(\theta)
$$

Then for fixed $\lambda, S_{\lambda}$ define a line bundle over $T_{\theta}$, because $\left|\tilde{\psi}_{\lambda}(\theta)\right\rangle=0$ nor $\left|\psi_{\lambda}(\theta)\right\rangle=0$ are generic (cf the discussion in the proof of theorem 5), and there is also periodicity with respect to $\theta$.

The mapping $\lambda \rightarrow S_{\lambda}$ is a homotopic deformation from the line bundle $\theta \rightarrow|\tilde{\psi}(\theta)\rangle$ to the line bundle $\theta \rightarrow\left|z\left(\theta_{1}, \theta_{2}\right)\right\rangle$. Their Chern index is constant and equal to $I$. 


\section{References}

[1] Thouless D J, Khomoto M, Nightingale M P and den Nijs M 1982 Quantized hall conductance in a twodimensional periodic potential Phys. Rev. Lett. 49

[2] Simon B 1983 Holonomy, the quantum adiabatic theorem and Berry's phase Phys. Rev. Lett. 51

[3] Bellissard J, van Elst A and Schulz-Baldes H 1994 The noncommutative geometry of the quantum Hall effect J. Math. Phys. 35 5373-451

[4] Thouless D 1994 Topological interpretations of quantum Hall conductance J. Math. Phys. 35 5362-72

[5] Colin de Verdière Y 1993 Fibrés en droites et valeurs propes multiples Sémin. Théorie Spectrale (Inst. Fourier, Grenoble) 92 9-18

[6] Avron J E, Seiler R and Simon B 1983 Homotopy and quantization in condensed matter physics Phys. Rev. Lett. 51

[7] Springsguth D, Ketzmerick R and Geisel T 1997 Hall conductance of Bloch electrons in a magnetic field Phys. Rev. B 562036

[8] Arnol'd V I 1972 Modes and quasimodes Funct. Anal. Appl. 6 94-101

[9] Lazutkin V F 1993 KAM Theory and Semiclassical Approximations to Eigenfunctions (Berlin: Springer) with an addendum by A I Shnirelman

[10] Arovas D P, Bhatt P N, Haldane F D M, Littlewood P B and Rammal R 1988 Localization, wavefunction topology, and the integer quantized hall effect Phys. Rev. Lett. 60

[11] Faure F 1994 Generic description of the degeneracies in Harper-like models J. Phys. A: Math. Gen. 27 $7519-32$

[12] Faure F and Parisse B 1998 Semiclassical study of the origin of quantized Hall conductance in periodic potentials J. Math. Phys. at press

[13] Dufour J-P, Molino P and Toulet A 1994 Classification des systemes integrables en dimension 2 et invariants des modeles de Fomenko. (Classification of integrable systems on two-dimensional manifolds and invariants in Fomenko's models) C. R. Acad. Sci., Paris 10 949-52

[14] Milnor J 1963 Morse Theory (Annals of Mathematics Studies 51) (Princeton, NJ: Princeton University Press)

[15] Griffiths P and Harris J 1978 Principles of Algebraic Geometry (New York: Wiley-Interscience)

[16] Helffer B and Sjöstrand J 1984 Multiple wells in the semiclassical limit-I Commun. Part. Diff. Eqns $9337-408$

[17] Wilkinson M 1984 Critical properties of electron eigenstates in incommensurate systems Proc. R. Soc. A 391

[18] Wilkinson M and Austin E J 1990 Phase space lattices with threefold symmetry J. Phys. A: Math. Gen. 23

[19] Voros A 1989 Wentzel-Kramers-Brillouin method in the bargmann representation Phys. Rev. A 40

[20] Saraceno M, Kurchan J and Leboeuf P 1989 Semiclassical approximation in the coherent state representation Phys. Rev. A 40

[21] Paul T and Uribe A 1993 A construction of quasi-modes using coherent states Ann. Inst. H. Poincaré Phys. Theor. 59 357-81

[22] Colin de Verdière Y and Parisse B 1994 Équilibre instable en régime semi-classique: I. Concentration microlocale Commun. Part. Diff. Eqns 19 1535-63

[23] Colin de Verdière Y and Parisse B 1994 Équilibre instable en régime semi-classique: II. Conditions de BohrSommerfeld Ann. Institut H. Poincaré Phys. Theor. 61 347-67

[24] Colin de Verdière Y and Parisse B 1999 Singular Bohr-Sommerfeld rules Commun. Math. Phys. 205 $459-500$

[25] Avron J E, Sadun L, Segert J and Simon B 1989 Chern numbers, quaternions, and Berry's phases in Fermi systems Commun. Math. Phys. 124 595-627

[26] Berry M V 1984 Quantal phase factors accompanying adiabatic changes Proc. R. Soc. 45

[27] Gudmundsson V and Gerhardts R R 1996 Phys. Rev. B 545223

[28] Schlosser T, Ensslin K, Kotthaus J P and Holland M 1996 Europhys. Lett. 33683

[29] Bargmann V 1961 On a Hilbert space of analytic functions and an associated integral transform Commun. Pure Appl. Math. 14 197-214

[30] Lebœuf P and Voros A 1990 Chaos-revealing multiplicative representation of quantum eigenstates J. Phys. A: Math. Gen. 231765

[31] Perelomov A 1986 Generalized Coherent States and their Applications (Berlin: Springer) 